\title{
Anhedonia is associated with reduced incentive cue related activation in the basal ganglia
}

\author{
Yu Sun Chung ${ }^{1}$ - Deanna Barch ${ }^{2}$
}

Published online: 24 June 2015

(C) Psychonomic Society, Inc. 2015

\begin{abstract}
Research has shown that reward incentives improve cognitive control in motivationally salient situations. Much previous work in this domain has focused on incentive cue-related activity in a number of brain regions, including the dorsolateral prefrontal cortex (DLPFC) and striatum. However, the more sustained changes in functional brain activity during task contexts with incentives have been relatively less explored. Here, we examined both the cue-related and sustained effects of rewards (i.e., monetary incentives) on cognitive control, with a particular focus on the roles of the DLPFC and striatum, using a mixed state-item design. We investigated whether variability in a reward-related trait (i.e., anhedonia) would modulate the sustained and/or the cue-related transient aspects of motivated cognitive control. Twentyseven healthy individuals performed a modified response conflict task (Padmala \& Pessoa, Journal of Cognitive Neuroscience, 23, 3419-3432, 2011) during scanning, in which participants were asked to categorize images as either houses or buildings with either congruent or incongruent overlaid words. Participants performed a baseline condition without knowledge of monetary incentives, followed by reward blocks with monetary incentives on some cued trials (reward
\end{abstract}

Electronic supplementary material The online version of this article (doi:10.3758/s13415-015-0366-3) contains supplementary material, which is available to authorized users.

Yu Sun Chung

yusun08@gmail.com

1 Department of Psychology, Washington University, St. Louis, MO, USA

2 Departments of Psychology, Psychiatry, and Radiology, Washington University, St. Louis, MO, USA cues) for fast and correct responses. We replicated previous work by showing increases in both sustained activity during reward versus baseline blocks and transient. cue-related activity in bilateral DLPFC and the basal ganglia. Importantly, healthy individuals with higher anhedonia showed less of an increase in trial-by-trial activity as a function of reward in the lateral globus pallidus. Together, our results suggest that reduced hedonic experience may be related to abnormality of reward cue-related activity in the basal ganglia.

Keywords Cognitive control $\cdot$ Motivation $\cdot$ Reward $\cdot$ Mixed fMRI design $\cdot$ Prefrontal cortex $\cdot$ Emotion

Accumulating evidence from both behavioral and neuroimaging studies consistently suggests that performance-contingent reward enhances cognitive control, as has been evidenced by the results from a variety of cognitive paradigms, including memory, attention, inhibition, and episodic memory (see Braver et al., 2014, for a recent literature review). This enhancing effect of rewards on cognitive control, sometimes referred to as "motivated cognitive control," is associated with changes in activity in a number of brain regions, including the lateral prefrontal cortex (PFC) and the ventral striatum (see, e.g., Boehler, Schevernels, Hopf, Stoppel, \& Krebs, 2014; Hollerman, Tremblay, \& Schultz, 2000; Ivanov et al., 2012; Rothkirch, Schmack, Deserno, Darmohray, \& Sterzer, 2014). Much of this research has focused on cue-related or transient changes in brain activation associated with the presentation of reward cues. However, motivational incentives can also increase brain activation in a sustained fashion (e.g., Engelmann, Damaraju, Padmala, \& Pessoa, 2009; Jimura, Locke, \& Braver, 2010), which has received less attention in the literature. As such, the purpose of this study was to 
examine both the sustained and transient effects of rewards (i.e., monetary incentives) on cognitive control, with a particular focus on the relative roles of the dorsolateral prefrontal cortex (DLPFC) and striatum. In addition, we investigated whether individual differences in a reward-related trait (i.e., anhedonia) would predict behavior or specific neural aspects of motivated cognitive control (i.e., sustained vs. transient effects).

\section{Transient/cue-related brain activity associated with motivational incentives}

The majority of the prior work on motivated cognitive control has focused on examining changes in behavioral performance and transient brain activation in response to external cues associated with reward value. A rich body of neuroimaging research has identified both cortical and subcortical brain regions (i.e., the lateral $\mathrm{PFC}$, striatum) that are responsive to representing, predicting, and updating reward value in motivationally salient contexts during cognitive performance (e.g., Boehler et al., 2011; Breiter \& Rosen, 1999; Dixon \& Christoff, 2012, 2014; Kouneiher, Charron, \& Koechlin, 2009; Krebs, Boehler, Roberts, Song, \& Woldorff, 2012; O’Doherty, 2004; Stoppel et al., 2011; Vassena et al., 2014; see Dixon \& Christoff, 2014, for a review). Single-unit studies in the lateral PFC with nonhuman primates have shown that the majority of neurons in the lateral PFC encode the representation of value-related information (Sakagami \& Watanabe, 2007). For example, when monkeys were trained to make go or no-go responses to the physical features of cue stimuli such as colors, most neurons in the lateral PFC showed differential visual responses to rewarding cues, regardless of the physical features of the cue stimulus (Watanabe \& Sakagami, 2007). In a similar vein, human neuroimaging and neurophysiological work has provided strong evidence that reward-predicting cues exert enhancing effects on cognitive control functions, thought to be supported by regions in the lateral PFC in a variety of cognitive control paradigms such as conflict processing (Krebs, Boehler, Appelbaum, \& Woldorff, 2013; Krebs, Boehler, \& Woldorff, 2010), working memory (Jimura et al., 2010; Pochon et al., 2002; Taylor et al., 2004), and context processing (Chiew \& Braver, 2013; Locke \& Braver, 2008). Many of these regions show transient increases in activations in response to cue information, indicating the potential for reward to be associated with upcoming performance.

These reward-predicting cues may be particularly relevant for modulating control function when there are competing stimulus dimensions, which often results in a high demand on cognitive control (e.g., Aupperle, Melrose, Francisco, Paulus, \& Stein, 2015; Padmala \& Pessoa, 2011). Behavioral and neuroimaging data in humans has shown that incentive cues can enhance cognitive control by decreasing conflict processing on reward trials (Krebs et al., 2010). For example, in neuroimaging work by Padmala and Pessoa (2011), healthy individuals performed a response conflict processing task, with reward trials cued by " $\$ 20$ " and no-reward trials by " $\$ 00$." Participants were instructed that correct and fast performance on " $\$ 20$ " trials would be rewarded. Consistent with prior studies showing enhancing effects of rewards on cognitive control (Engelmann, Damaraju, Padmala, \& Pessoa, 2009; Small et al., 2005), these researchers found that cue-related neural responses on reward trials were increased in several fronto-parietal regions, as well as in the ventral striatum and caudate. Also, these researchers found that conflict-related responses in the medial PFC on reward trials were reduced as compared to on no-reward trials.

\section{Sustained brain activations associated with motivational incentives}

Transient brain responses to cues indicating the potential for reward may not be the only mechanism that links incentive information to enhanced cognitive control. It is possible that information about potential rewards may change cognitive processing and brain activity during the entire task in a more sustained fashion. A more recent line of research has examined state-dependent reward context effects on cognitive control, providing evidence for the presence of motivation-related "state" effects on cognitive function, as evidenced by increased sustained activations across blocks of trials with incentive information (Engelmann et al., 2009; Jimura et al., 2010; Locke \& Braver, 2008). For example, in a study by Engelmann et al. (2009) using a Posner-type task in which cues indicated the location of the face target stimulus, motivation was manipulated in a blocked fashion by varying the valence (e.g., winning, avoiding loss) and magnitude of the rewards associated with task performance (e.g., winning $\$ 1$ or $\$ 4$, or avoid losing $\$ 2.5$ or $\$ 0$ ). They found that in several regions in a fronto-parietal attentional network (i.e., the posterior intraparietal sulcus, middle frontal gyrus, the caudate, and putamen), cue-related responses were modulated by the incentive values. Importantly, they also found that several regions thought to be involved in the control of attention (e.g., the intraparietal sulcus and middle frontal gyrus) showed increased sustained activations across the course of blocks with greater incentive values. These results suggest that the enhancing effects of rewards on cognitive control can be evidenced in at least two ways: by (1)increases in cue-related responses, and (2)increases in sustained responses.

In a similar vein, Locke and Braver (2008) had healthy adults perform a goal maintenance task under baseline, reward, and penalty conditions during scanning. These researchers found an increase in sustained activations during reward blocks in a network of cognitive control regions including the right lateral PFC (i.e., DLPFC and ventrolateral 
PFC) and the parietal cortex, as well as an improvement in task performance on trials in the reward blocks. Importantly, work by Jimura et al. (2010) extended the prior findings by showing that during reward blocks, better performance was seen even on trials on which people could not earn rewards. Specifically, when healthy individuals completed a working memory task under no-reward versus reward contexts, they showed faster performance on trials for which they could earn reward than on trials in a baseline condition (e.g., Locke \& Braver, 2008; Padmala \& Pessoa, 2011). Interestingly, their performance was better even on trials in the reward condition for which they could not earn reward, as compared to the baseline condition, an effect Locke and Braver referred to as a reward context effect. Importantly, this behavioral incentive context effect was associated with an increase in the right DLPFC (BA 9/46) activation that was sustained across both reward and nonreward trials in the task blocks (Jimura et al., 2010).

\section{Incentives and the dual-mechanisms-of-control framework}

To explain these findings, Jimura et al. (2010) invoked a theoretical framework of cognitive control developed by Braver and his colleagues (Braver, 2012; Braver, Gray, \& Burgess, 2007), referred to as the dual-mechanisms-ofcontrol theory. This theory postulates that cognitive control can be supported by at least two complementary mechanisms. The first is proactive control, which is the engagement of control mechanisms, including support for task-relevant goals and tasks sets, prior to and in anticipation of the need to implement them. The second is reactive control, which is the triggering of control mechanisms, such as the retrieval of task sets or goals, when conflict or difficulty in processing is encountered. This theory suggests that both the behavioral improvements seen on neutral trials in reward contexts and the increase in sustained activation in the DLPFC might reflect a role of the right lateralized DLPFC in the preparatory, proactive control used to integrate reward-related information with cognitive goal representations. That is, even the knowledge that it is possible to gain rewards may enhance cognitive control, even when the current trial does not contain a specific incentive cue. This may occur by facilitating the representation of task-relevant information (Niv, 2007; reviewed in Braver et al., 2014), which may be reflected in enhanced sustained activity, as well as by increased cue-related responses, in regions such as the DLPFC. Interestingly, Jimura et al. (2010) also found that such increases in cue-related responses (putatively reflecting enhanced proactive control) were accompanied by reduced probe-related responses in the same DLPFC region, potentially reflecting a decreased need for reactive control.

\section{Individual-difference factors relating to motivated cognitive control}

Importantly, providing rewards or incentives does not lead to changes in behavioral performance and brain activity in all individuals. A growing body of research suggests that individual differences in reward-related sensitivity may modulate behavioral and neural responses to either primary (e.g., food) or secondary (i.e., monetary incentives) rewards (e.g., Beaver et al., 2006; Cooper, Duke, Pickering, \& Smillie, 2014; Jimura et al., 2010; Locke \& Braver, 2008). Specifically, several studies have reported associations between individual differences in reward-related personality traits and reward-related neural activations (e.g., Beaver et al., 2006; Cohen, Young, Baek, Kessler, \& Ranganath, 2005; Cooper et al., 2014; Jimura et al., 2010; Locke \& Braver, 2008). For example, Beaver et al. (2006) found that individual differences in Behavioral Inhibition and Behavioral Approach Systems (BIS/BAS) drive scores (i.e., items asking about the pursuit of goals) were significantly associated with neural responses to appetizing relative to bland foods in the ventral striatum and orbitofrontal cortex. Cohen et al. (2005) found that when participants received immediate monetary rewards during a gambling task, people higher in extraversion showed a greater magnitude of neural response related to reward receipt versus no reward in the right medial orbitofrontal cortex, amygdala, and right nucleus accumbens. More recent work by Jimura et al. (2010) also showed positive associations between sustained DLPFC activations during reward context and reward sensitivity from the BAS. Thus, personality traits related to reward drive and sensitivity may be factors for understanding individual differences in incentive effects on cognitive control.

However, another less explored individual-difference factor related to reward processing is anhedonia. Anhedonia is defined as a reduction in the ability to experience pleasure. Experiencing rewards as positive or pleasurable maybe a critical factor that induces approach behavior toward goals and positive emotional states (e.g., as is reviewed in Gorwood, 2008). Even nonclinical populations show individual differences in anhedonia (see, e.g., Franken, Rassin, \& Muris, 2007; Harvey, Pruessner, Czechowska, \& Lepage, 2007). Individuals who experience rewards as being less pleasurable may be less "motivated" to modulate their behavior in order to enhance the likelihood of achieving such rewards. As such, individuals who self-report higher levels of anhedonia may show less improvement in cognitive control as a function of reward, and potentially less modulation for incentive-related brain activity, though it is less clear whether anhedonia may influence transient or sustained modulation of brain activity, or both. 


\section{Hypotheses of the present study}

The purpose of this study was first to replicate prior work examining the neural mechanisms that mediate an enhancing effect of rewards on cognitive control, by examining sustained as well as incentive cue-related effects on cognitive control using a mixed state-item fMRI design. We focused on the DLPFC and the striatum, given prior research suggesting their involvement in mediating the influence of rewards on cognitive control. We modified a response conflict processing task originally developed by Padmala and Pessoa (2011) to fit in a mixed state-item design. The state-item fMRI design enabled us to examine sustained context-dependent effects and transient reward-related cue effects in the same study. Participants first performed baseline conditions without knowledge of the potential for incentives in future blocks. Participants then performed additional reward blocks on which they were told that they could win money on some trials (rewarded trials) by performing quickly and accurately. This variant of the paradigm enabled examinations of (1)reward context effects, by comparing performance and brain activity during the baseline versus the reward context, and (2)reward cue effects, by comparing performance during reward versus no-reward trials within reward blocks, as well as trials during the baseline blocks. We predicted that motivational states induced by reward contexts would produce greater sustained activity in the DLPFC than would those in nonincentive baseline blocks. We also predicted that incentive cues would generate increased transient neural activity in both reward-related cortical and subcortical regions.

Second, we wanted to test the hypotheses that individual differences in self-reported anhedonia would moderate the influence of rewards on cognitive control, with higher levels of self-reported anhedonia being associated with less of an improvement in performance as a function of reward, as well as with less of an increase in the activity of the DLPFC and/or striatum in response to reward information, in terms of either sustained or transient activation (or both). To test these hypotheses, the present study included two measures of trait anhedonia: the Snaith-Hamilton Pleasure Scale (Snaith et al., 1995) and the Social and Physical Anhedonia Scales (Chapman \& Chapman, 1978; Eckblad, Chapman, Chapman, \& Mishlove, 1982).

\section{Method}

\section{Participants}

A total of 28 healthy adults participated in the present study. One participant was not included in final analyses because the person failed to pass the fMRI quality control (described below). Thus, 27 healthy adults were included in final analyses (see Table 1 for the participant characteristics). Originally, we had planned to include 30 participants, to achieve $80 \%$ power to detect a medium effect size (Cohen's $d=0.5$ ), in order to replicate previous findings of changes in state-related activity between task blocks ( $d=0.5$ in a paired $t$ test) and in cuerelated activity (effect size $f=0.15$; repeated measures analysis of variance [ANOVA] with one within-subjects factor, Trial Type). Due to practical constraints, we were able to include a sample size of 27 , which provided $75 \%$ power to detect these effect sizes. Also, this sample size provided at least $68 \%-86 \%$ power to detect correlation coefficients ranging from .4 to .5 or greater.

All participants had no personal or family history of psychiatric or neurological disease. All participants were recruited through the Conte Center for the Neuroscience of Mental Disorders at Washington University in St. Louis and provided written informed consent. The study protocol was approved by the Washington University Human Research Protection Office. Participants received a maximum of $\$ 20$ reward money that depended on their correct and fast behavioral performance, in addition to money for completing the experiment $(\$ 25 / h)$.

\section{Procedure}

The study structure consisted of two sessions, one behavioral and one neuroimaging. In the behavioral session, participants completed several individual-difference questionnaires, described below, and a demographic form. During the neuroimaging session, they performed a modified response conflict processing task inside the scanner. They had two practice sessions, one before baseline blocks and one before the reward blocks, inside the scanner, to make sure that they were familiar with the task. At the end of the neuroimaging session,

Table 1 Participants characteristics and individual difference measures

\begin{tabular}{ll}
\hline Variables & Healthy Adults $(N=27)$ \\
\hline Age (years) & $35.56(8.61)$ \\
Gender (\% male) & $55.6 \%$ \\
Race (\% Caucasian) & $29.6 \%$ \\
Smoking status (\%Smokers) & $37.0 \%$ \\
Handedness (\% right) & $92.6 \%$ \\
Highest Parental Education (years) & $14.11(1.73)$ \\
Education (years) & $14.51(1.86)$ \\
Individual-Difference Measures & \\
$\quad$ Snaith-Hamilton Pleasure Scale & $52.70(3.27)$ \\
$\quad$ Beck Depression Inventory & $2.14(3.55)$ \\
Chapman Social Anhedonia & $7.96(6.03)$ \\
Chapman Physical Anhedonia & $9.81(5.26)$ \\
\hline
\end{tabular}

Numbers represents means and $S D$ s 
participants completed a postscan questionnaire to measure self-reported motivation and task difficulty during the task.

\section{Stimuli and task paradigm}

A mix of images-plus-words was used for the stimuli. The images were of either a house or a building, and each image was overlaid with a word to create congruent, incongruent, and neutral trials. For example, a congruent trial was one in which an image was presented with a matching word (e.g., a house picture with "HOUSE" below it, or a building picture with "BLDNG"). However, an incongruent trial was one in which an image was presented with a conflicting word (e.g., a house picture with "BLDNG" or a building picture with "HOUSE"). Neutral trials were ones in which an image was presented with "XXXXX."

The task was a variant of a previously validated reward task paradigm (Padmala \& Pessoa, 2011) that has revealed transient cue-related enhancing effects of monetary incentives on conflict processing in healthy individuals. We modified this task to use a mixed state-item fMRI design, allowing us to examine both the sustained context-dependent effects of reward and the transient cue-related effects of rewards. We used the recommendations for mixed state-item fMRI design from Petersen and Dubis (2012; see Fig. 1a). The fMRI task paradigm consisted of two baseline and four reward runs, with 54

(a) Mixed State-Item fMRI Design

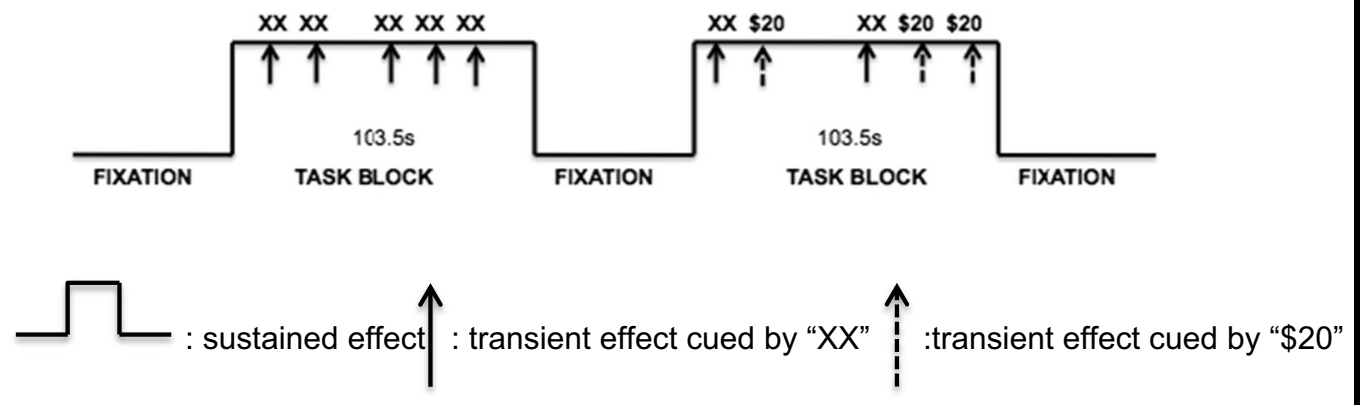

(b) Task Stimuli

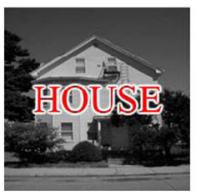

House Congruent

(c) Example Trial of Baseline-Context Block

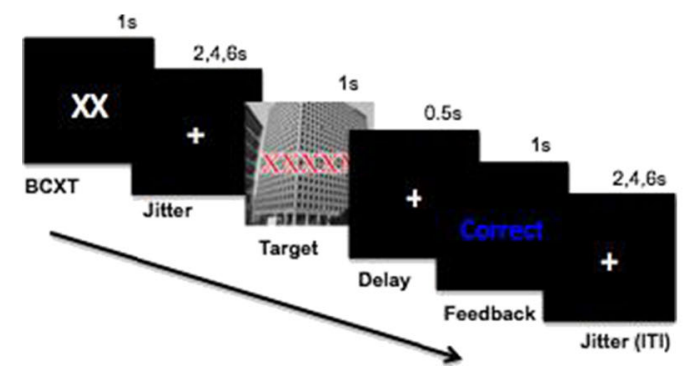

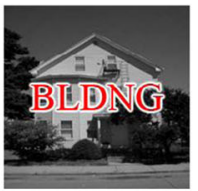

House Incongruent

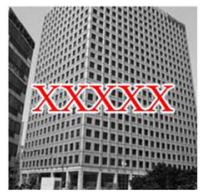

Building Neutral (d) Example Trial of Reward Blocks

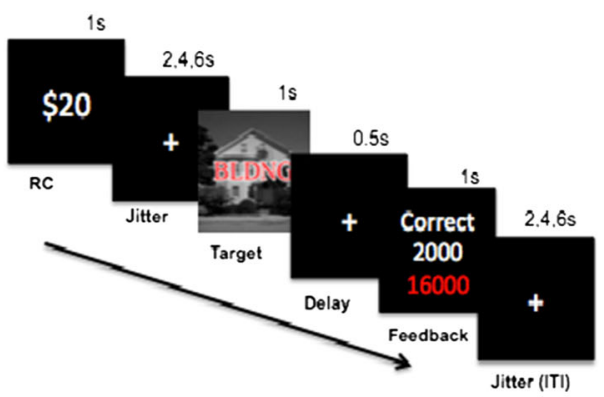

Fig. 1 Mixed state-item fMRI design of our response conflict task 
trials per each trial type (i.e., baseline context, reward cue, and reward context), resulting in a total of 162 trials. During the task, participants were instructed to focus on the image and ignore the overlaid letters, and to categorize each picture by pressing a "1" for a house image or " 2 " for a building image.

As is presented in Fig. 1c, the baseline blocks consisted of two runs in which three different types of trials (i.e., congruent, incongruent, and neutral) were intermixed, with 18 trials per trial type (total of 54 trials). Each task block started with a start cue, "TASK," and ended with an end cue, "DONE," each presented for $2 \mathrm{~s}$. After each start cue was a jittered period ranging from 0 to $4 \mathrm{~s}$. During the baseline blocks, each trial started with an "XX" cue for $1 \mathrm{~s}$, with prior instruction to the participants indicating that these cues were not relevant to the task. Then a jittered fixation period occurred, ranging from 2 to $6 \mathrm{~s}$ before the onset of the stimuli, to allow for estimates of event-related responses to the cues. The target stimulus was next presented for $1 \mathrm{~s}$, which was followed by a delay of $0.5 \mathrm{~s}$, during which time the participants responded. Participants were then provided with visual feedback indicating whether their performance was correct or incorrect, for $1 \mathrm{~s}$. Finally, there was an intertrial interval (ITI) that varied between 2, 4, and $6 \mathrm{~s}$.

After the two baseline runs, participants performed four additional runs, for which they were instructed that they could win money on some trials for their correct and fast responses, as is presented in Fig. 1d. The reaction time (RT) threshold to determine "fast" responses was set individually on the basis of the median correct RT for the second baseline run. During reward runs, half of the trials were preceded by a " $\$ 20$ " cue (reward cue: RC), indicating that a fast and correct response would be rewarded by 2,000 points, or by an "XX" cue (reward context: RCXT), indicating that zero points would be possible on the trial. A total of 108 trials were presented, with approximately equal numbers of congruent, incongruent, and neutral trials. After the target stimulus, participants received feedback regarding the reward points they had earned on that trial, as well as their cumulative earning in points. The accumulated points were converted into real money at the end of the experiment (with a maximum of \$20).

\section{fMRI data acquisition and processing}

Imaging data were acquired on a 3-T Siemens TM TRIO system with a 12-channel head coil. Both structural and functional images were acquired every scanning session. Highresolution MPRAGE T1 images (echo time $[\mathrm{TE}]=2.98 \mathrm{~ms}$, repetition time $[\mathrm{TR}]=2,300 \mathrm{~ms}, 160$ slices, $1.0 \times 1.0 \times$ $1.2 \mathrm{~mm}$ voxels $)$ and $\mathrm{T} 2$ images $(\mathrm{TE}=84 \mathrm{~ms}, \mathrm{TR}=7$, $000 \mathrm{~ms}$, 33 slices, $2.0 \times 1.0 \times 4.0 \mathrm{~mm}$ voxels) were acquired, to be registered and transformed to a standardized atlas space (Koch et al., 2010; Talairach \& Tournoux, 1988) using a 12dimensional affine transformation (Gradin et al., 2011;
Woods, Cherry, \& Mazziotta, 1992). Functional images were collected in six runs of 214 frames each, using an asymmetric spin-echo echo-planar sequence $(\mathrm{TR}=2,000 \mathrm{~ms}, \mathrm{TE}=27 \mathrm{~ms}$, field of view $=256 \mathrm{~mm}$, flip $=90^{\circ}, 33$ slices). The functional images were acquired parallel to the anterior-posterior commissure plane with $4-\mathrm{mm}^{3}$ isotopic voxels. Visual stimuli were projected using the E-Prime software, running on a Dell Inspiron laptop. Each stimulus was projected to participants with an LCD projector onto a screen located behind the scanner. A fiber-optic, light-sensitive keypress interfaced with the E-Prime button box was used to record participants' behavioral performance inside the scanner.

All imaging data were preprocessed and analyzed using inhouse Washington University software (the FIDL analysis package: www.nil.wustl.edu/ fidl/). The first four images of each run were discarded to allow for signal stabilization. The functional-imaging data preprocessing included (1)correction for slice-dependent time shifts; (2)removal of the first four images of each run; (3)elimination of odd/even slice intensity differences due to interpolated acquisition; (4)realignment of the data to compensate for rigid-body motion; (5) normalization of image intensity to a whole-brain mode value of 1,000 ; (6)registration of the 3-D structural volume (T1) to the atlas template in the Talairach coordinate system, using a 12-parameter affine transform and resampling to a 1-mm cubic representation (Buckner et al., 2004; Ojemann et al., 1997) ; (7) coregistration of the 3-D fMRI volume to the T2, and the T2 to the structural image; (8) transformation of the fMRI data to a $3 \times 3 \times 3 \mathrm{~mm}$ voxel atlas space, using a single affine 12 parameter transform; and (9) spatial smoothing using a 6-mm full-width at half-maximum Gaussian filter. We assessed head movement during scanning by using the output of the rigidbody rotation and translation algorithm. The translations and rotations in the $x, y$, and $z$ planes across frames and the total root-mean-square (RMS) linear and angular precision measures were calculated for each run. If the standard deviation of the RMS movement exceeded 20, the BOLD runs were not included in the analysis [RMS/frame, as mean $(S D)$ : 27 participants $=0.15(0.07)]$.

\section{Self-reports of anhedonia}

Participants completed the Snaith-Hamilton Pleasure Scale (SHPS; Snaith et al., 1995), which consists of 14 items that assess hedonic tone with a 4-point Likert scale $(1=$ definitely disagree, $4=$ definitely agree). Thus, low scores represent the absence of hedonic tone (i.e., anhedonia). The SHPS has demonstrated high internal consistency, good test-retest reliability, and good convergent validity and discriminant validity (Franken et al., 2007). Participants also completed the Revised Social Anhedonia Scale (Eckblad et al., 1982) and the Revised Physical Anhedonia Scale (Chapman \& Chapman, 1978). These scales measure the self-reported 
ability to experience pleasure from either social or physical stimuli using true/false questions. High scores represent high levels of anhedonia. Both the Social and Physical Anhedonia Scales have shown good internal consistency and test-retest reliability (Chapman, Chapman, \& Kwapil, 1995).

\section{Data analysis}

Behavioral data Repeated measures ANOVAs were conducted on the median RTs for correct trials and accuracy, with the within-subjects factors Reward (BCXT, RC, RCXT) and Trial Type (congruent, incongruent, and neutral). Post-hoc paired $t$ tests followed, used to determine the source of significant interactions. A behavioral index of reward context effects was estimated by subtracting the RT on RCXT trials, cued by "XX," from that on BCXT trials, with the same cue, "XX." An index of reward cue effects was computed by subtracting the RT on RC trials, cued by " $\$ 20$," from that on RCXT trials, cued by "XX" within the same reward conditions. These behavioral indices of reward context and cue effects were used in a Pearson correlation analysis to examine the associations between individual differences in anhedonia trait and behavioral enhancements of rewards on cognitive tasks.

fMRI data We used the recommendations for mixed stateitem fMRI design from Petersen and Dubis (2012; see Fig. 1a). According to these recommendations, we simultaneously coded both sustained and transient cues, and targetrelated brain activity within the same general linear model (GLM). Specifically, in terms of modeling transient events, we used unassumed hemodynamic responses with eight time points, considering evidence that any deviation from the assumed shape using canonical waveforms can be misattributed to the sustained activation component (Miezin, Maccotta, Ollinger, Petersen, \& Buckner, 2000; Visscher et al., 2003). The unassumed GLMs enabled us to obtain one parameter for every time point following stimulation in each event type modeled (Manoach, Greve, Lindgren, \& Dale, 2003; Ollinger, Corbetta, \& Shulman, 2001a; Ollinger, Shulman, \& Corbetta, 2001b). Thus, we could test differences between the event types of interest by focusing on regions showing interactions with time point, due to our use of unassumed GLMs. As is reviewed by Petersen and Dubis (2012), the mixed state-item design enables one to dissociate sustained and transient effects with the assumption that event-related trial-by-trial effects should decay back to baseline during the ITI, whereas the state effects should remain sustained during the entire task block. Thus, the sustained effects (i.e., baseline and reward blocks) were modeled by boxcar functions lasting the length of the task block, using an assumption of a fixedshape response of long duration (Fischl et al., 2002). Eventrelated cue and target effects for each trial type were analyzed separately by estimating the values of eight time point regressors (starting at trial onset) within the hemodynamic response epoch, leading to a 16-s estimate (TR $2 \mathrm{~s}$, eight scanning frames) using unassumed hemodynamic response shapes. Thus, the model included two regressors for the sustained effects (baseline and reward context), three regressors for the cue types (BCXT "XX," RC “\$20," and RCXT “XX”), and two regressors for the start and end cues, respectively, during the cue phase. Considering prior research suggesting that the effect of rewards on cognitive control can be reflected in changes to the cues, either reward or no reward in reward contexts (e.g., Jimura et al., 2010), and not only in sustained activity, we used the same cue, "XX," in the baseline and reward contexts and modeled the regressor for BCXT in order to test this possibility. During the target phase, nine regressors for the combination of each cue type with each trial type (congruent, incongruent, and neutral) were included.

We used voxel-wise paired $t$ tests to analyze the sustained estimates, with Reward (baseline, reward) as the withinsubjects factor. For cue-related activity, we conducted a voxel-wise repeated measures ANOVA with Reward (BCXT, RCXT, RC) and Time Point (the eight time frame estimates for the hemodynamic response) as within-subjects factors. We focused on regions showing interactions with time point, due to our use of unassumed GLMs. Post-hoc ANOVAs and $t$ tests were performed within all significant regions identified by the ANOVAs, as we described above. For these post-hoc analyses, we extracted the mean percent signal change across each region for each time point out of the eight estimated time points, to visualize the pattern of activity. For the statistical analyses, we focused on Time Point 4, because this time point encompassed $7-8 \mathrm{~s}$ after stimulus onset, which corresponded to the initial peak in a stereotyped hemodynamic responses, unconfounded by sustained activity. This was done for each applicable cue type and trial type effect. We then conducted post-hoc paired $t$ tests to compare the three trial types, to parse the significant cue-related and condition-related effects.

For target-/receipt-related activation, we conducted two separate repeated measures ANOVAs. The first ANOVA ignored trial type (i.e., congruent, neutral, incongruent) and compared activation in the target phase across trials that could or could not earn reward in either the baseline or the reward blocks (i.e., Reward: BCXT, RCXT, or RC). Post-hoc paired $t$ tests at Time Point 4 (i.e., BCXT-RC, RC-RCXT, BCXTRCXT) were conducted to follow up on any significant effects. To attempt to replicate previous findings from prior work by Padmala and Pessoa (2011), another repeated measures ANOVA also included trial type (i.e., congruent, neutral, incongruent) and compared activation in the target phase across trials with activation in the reward context when participants could or could not earn reward, with Reward Context (i.e., BCXT, RC), Trial Type (i.e., congruent, neutral, incongruent trials), and Time Point as within-subjects factors. 
DLPFC and basal ganglia (BG) a priori masks (see Supplemental Fig. 1) We constrained our analyses to a priori masks within the DLPFC and BG, given the involvement of these regions in reward processing (see, e.g., Jimura et al., 2010; Locke \& Braver, 2008). We used anatomically defined masks of voxels within the DLPFC (Rajkowska \& GoldmanRakic, 1995) and BG (Wang et al., 2008). Voxel-by-voxel neuroimaging analyses were conducted within these masks. All statistical activation maps from these masks were corrected for multiple comparisons using combined $p$-value and cluster thresholds, determined by the AlphaSim program in the AFNI software package. The DLPFC mask (Brodmann's areas 9 and 46) included both left and right middle and superior frontal gyri according to anatomical landmarks (Rajkowska \& GoldmanRakic, 1995). For the DLPFC mask, we used as thresholds a $z$ value of 2.05 and 13 voxels. The BG mask was based on Wang et al. (2008) and was generated by combining the caudate, nucleus accumbens, putamen, and globus pallidus together. We applied a $z$ value of 2.05 and a 14-voxel threshold for the BG mask. Then we used information about the centroid of activation in these identified subregions to label them as a specific area. For any follow-up analyses to identify the sources of significant effects and further correlations analyses, as described above, we extracted the average of the BOLD response values of the voxels within the identified subregions within the mask regions and imported them into SPSS.

Individual difference analyses We examined whether individual differences in anhedonia predicted behavior and/or the degree of brain activations as a function of rewards in the regions of interest (ROIs) that showed sustained reward and transient cue effects within each DLPFC and BG mask. We examined whether either of the following individualdifference measures predicted behavior, the magnitude of sustained brain activation during reward versus baseline contexts, or transient activation during RC versus RCXT trials: (1) total SHPS scores and (2)a composite score of the Chapman social and physical anhedonia scores ( $z$-scored and then combined into one composite score). The brain-personality trait correlations were corrected by using the same smallvolume procedures described above.

\section{Results}

\section{Behavioral data}

\section{Reaction time (Fig. 2a)}

The repeated measures ANOVA indicated significant main effects of reward $\left[F(2,52)=18.20, p<.001, \eta_{\mathrm{p}}{ }^{2}=.41\right]$ and trial type $\left[F(2,52)=21.00, p<.001, \eta_{\mathrm{p}}{ }^{2}=.45\right]$, but no significant Reward $\times$ Trial Type interaction $[F(4,104)=$ $\left.1.36, p=.25, \eta_{\mathrm{p}}{ }^{2}=.05\right]$. The main effect of reward (BCXT,
RCXT, RC) reflected faster performance on RCXT than on BCXT trials $\left[F(1,26)=4.09, p=.05, \eta_{\mathrm{p}}{ }^{2}=.14\right]$, as well as faster performance on RC than on RCXT trials $[F(1,26)=$ $\left.26.55, p<.001, \eta_{\mathrm{p}}{ }^{2}=.50\right]$. The main effect of trial type indicated slower responses on incongruent than on congruent trials $\left[F(1,26)=28.92, p<.001, \eta_{\mathrm{p}}{ }^{2}=.53\right]$ and neutral trials $[F(1$, 26) $\left.=12.75, p<.002, \eta_{\mathrm{p}}{ }^{2}=.33\right]$, and slower RTs on neutral than on congruent trials $\left[F(1,26)=17.41, p<.001, \eta_{\mathrm{p}}{ }^{2}=.40\right]$.

Figure 2a seemingly shows that the magnitude of interference effects might be greater in the RC condition, even though the overall RTs were faster there. We conducted a paired $t$ test comparing the interference effect (i.e., incongruent- neutral) on the $\mathrm{RC}$ as compared to the BCXT blocks. The interference effects were not significantly different during RC (mean = $38.64, S E M=12.64)$ than during BCXT (mean $=17.79$, $S E M=8.96)$ blocks $[t(26)=1.58, p=.13]$.

Accuracy (see Fig. 2b) The analogous ANOVA on the error data indicated a significant main effect of trial type $[F(2,52)=$ $\left.13.91, p<.001, \eta_{\mathrm{p}}{ }^{2}=.35\right]$, reflecting more errors on incongruent than on congruent trials $\left[F(1,26)=16.36, p<.001, \eta_{\mathrm{p}}{ }^{2}\right.$ $=.38]$. We observed no significant main effect of reward $[F(2$, $\left.52)=1.20, p=.31, \eta_{\mathrm{p}}{ }^{2}=.04\right]$ and no significant interaction of reward and trial type $\left[F(4,104)=0.83, p=.51, \eta_{\mathrm{p}}{ }^{2}=.03\right]$. Given the lack of a significant main effect of reward on the error data, further analyses were focused on the RT data.

Behavioral measures Neither the behavioral indices of reward context nor those of the reward cues were significantly correlated with individual differences in the anhedonia measures.

\section{Neuroimaging results}

Sustained effects of reward As is presented in Table 2 and Fig. 3, the analyses revealed a significant main effect of reward in bilateral DLPFC regions as well as in the dorsal striatum, with greater activity during reward as compared to baseline blocks.

Relationship between sustained effects of reward and anhedonia Individual differences in responses to the Chapman scales and SHPS were not significantly associated with the magnitude of sustained activation in the comparison of reward to baseline in the ROIs described above that showed significant sustained activation (all $p \mathrm{~s}>.10$ ).

Cue-related effects of reward Regions in the bilateral DLPF $\mathrm{C}$, lateral globus pallidus, and caudate body displayed significant interactions between reward (RC, RCXT, BCXT trials) and time point (see Table 3). Post-hoc paired $t$ tests at Time Point 4 (i.e., RC-RCXT, RC-BCXT, RCXT-BCXT) were conducted to determine the source of the effect of reward. Consistent with prior work showing the involvement of the DLPFC in reward-related effects (e.g., Jimura et al., 2010; 


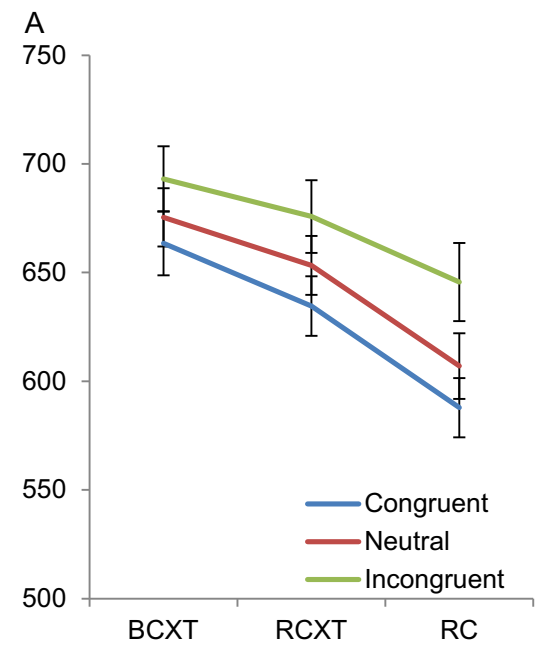

Fig. 2 Behavioral data: Reaction times and error data

Pochon et al., 2002; Watanabe, 2007), bilateral DLPFC showed increased cue-related activation on $\mathrm{RC}$ relative to both RCXT and BCXT trials. Similarly, several regions in the left lateral globus pallidus and left caudate body and head also showed increased cue-related activity during RC as compared to RCXT and BCXT trials (right lateral globus pallidus) (see Fig. 4 for example time courses). However, we did not see any regions in DLPFC or striatum that showed greater cue-related activation on RCXT than on BCXT trials (all $p \mathrm{~s}>.05$ ).

\section{Relationship between cue-related effects of reward and} anhedonia Individuals reporting greater anhedonia on the Chapman scales showed less cue-related activation as a function of reward-predicting cues in the lateral globus pallidus $(r$ $=-.54, p=.003$; see Fig. $5 \mathrm{~A}$ for a scatterplot), an effect that passed Bonferroni correction (.05/6). We saw a similar relationship in this region to self-report of hedonic tone on the SHPS, with greater hedonic tone being associated with greater cue-related activation as a function of reward-predicting cues in the lateral globus pallidus ( $r=.45, p=.02$; see Fig.5B), though this correlation did not pass Bonferroni correction.

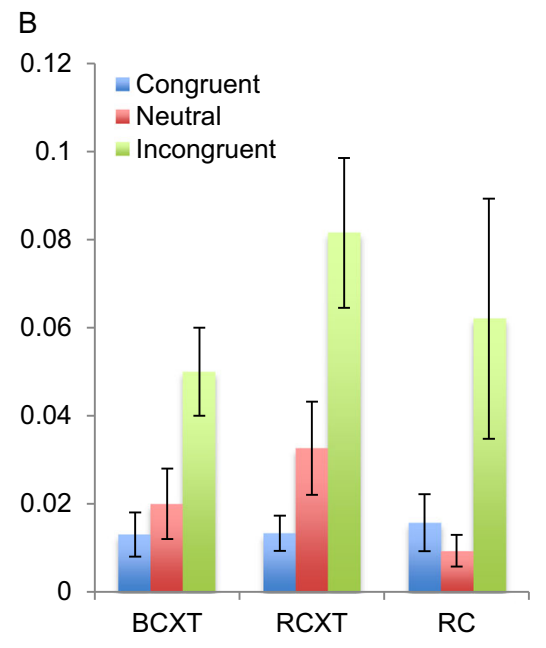

Effects of reward context on target-related activation Regions in the DLPFC and the BG that displayed a significant reward context effect showed two patterns of results (see Table 4). In the top panel of Table 4, regions in the DLPFC and the BG show reduced target-related activation on RC relative to RCXT or BCXT trials. For example, in the medial portion of the right DLPFC (BA 9: $x=26, y=37, z=29$ ), target-related activation on RC and RCXT trials was significantly reduced relative to BCXT trials. Interestingly, in several subcortical regions, such as the lateral globus pallidus, targetrelated activity at Time Point 4 did not differ among rewardrelated types. However, the source of the significance effect was observed at Time Point 7, with a greater degree of deactivation on RC than on BCXT and RCXT trials (see Fig. 6 for time courses from the DLPFC and BG regions displaying Reward Context $\times$ Time Point effects in the target phase). In the bottom panel of the same table, a different set of larger, more lateral, and more posterior regions in the DLPFC showed greater activation on $\mathrm{RC}$ relative to $\mathrm{BCXT}$ trials (see Supplemental Fig. 2 for the time courses of each region in the DLPFC).

Table 2 Sustained effects of reward

\begin{tabular}{|c|c|c|c|c|c|c|c|c|}
\hline \multirow[t]{2}{*}{ Analysis } & \multirow[t]{2}{*}{ BA } & \multirow[t]{2}{*}{ Region of Activation } & \multirow[t]{2}{*}{ Cluster Size (voxels) } & \multicolumn{3}{|c|}{ Talairach Coordinates } & \multirow[t]{2}{*}{$Z$} & \multirow[t]{2}{*}{ Activation Pattern $^{a}$} \\
\hline & & & & $x$ & $y$ & $z$ & & \\
\hline \multirow[t]{3}{*}{ DLPFC } & 46 & Middle frontal gyrus & 18 & 43 & 29 & 21 & 2.81 & $\mathrm{R}>\mathrm{B}$ \\
\hline & 9 & Middle frontal gyrus & 149 & 39 & 14 & 31 & 3.20 & $\mathrm{R}>\mathrm{B}$ \\
\hline & 9 & Precentral gyrus & 60 & -38 & 10 & 30 & 2.95 & $\mathrm{R}>\mathrm{B}$ \\
\hline \multirow[t]{3}{*}{ Basal ganglia } & & Right dorsal striatum & 57 & 23 & 7 & 4 & 3.21 & $\mathrm{R}>\mathrm{B}$ \\
\hline & & Left dorsal striatum & 19 & -23 & 7 & 5 & 2.67 & $\mathrm{R}>\mathrm{B}$ \\
\hline & & Left dorsal striatum & 19 & -18 & 2 & 16 & 2.79 & $\mathrm{R}>\mathrm{B}$ \\
\hline
\end{tabular}

$Z$ values represent mean activation across the region

$D L P F C$ dorsolateral prefrontal cortex, $R$ reward, $B$ baseline 

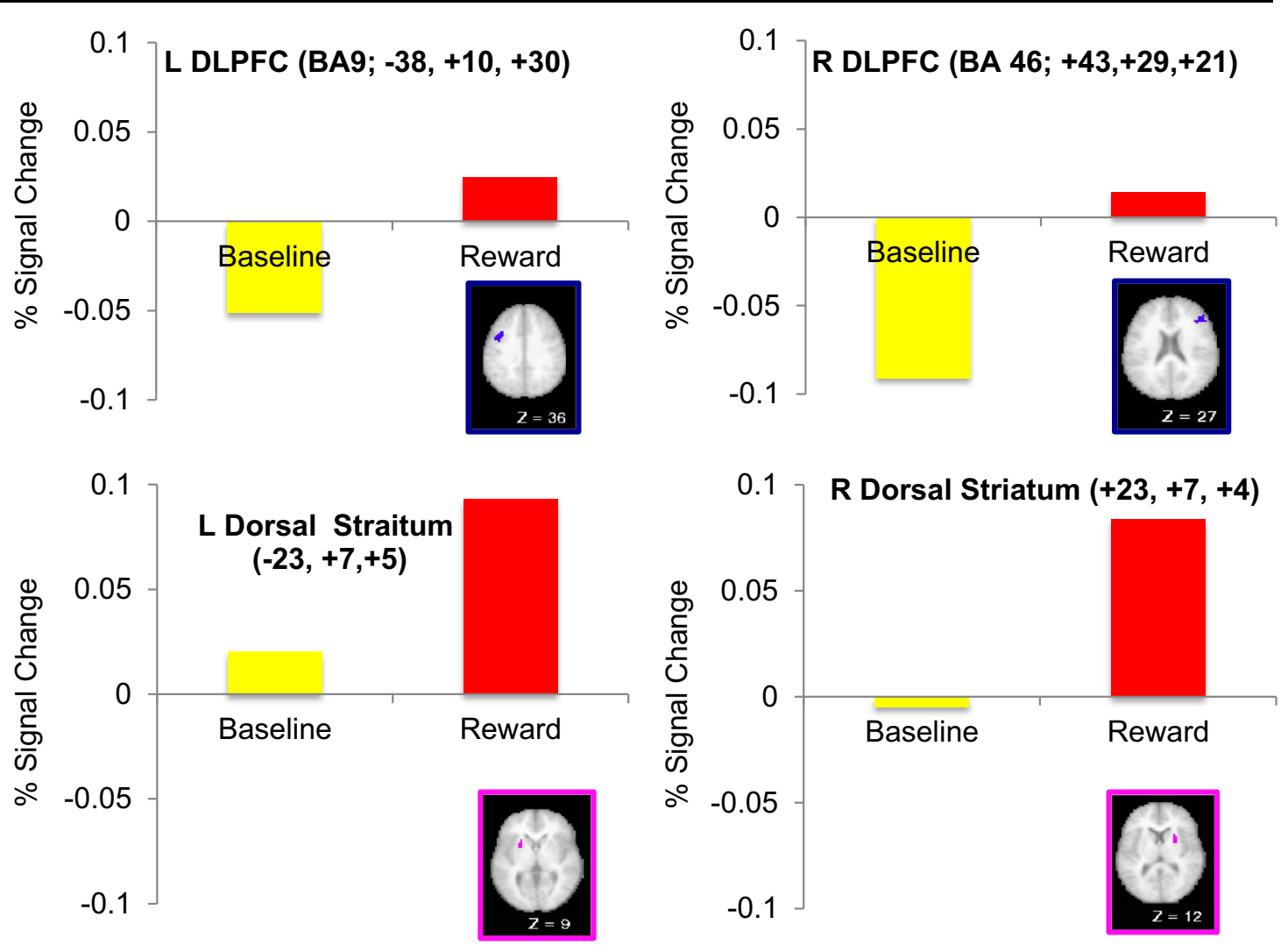

Fig. 3 Regions displaying sustained effects of reward

Effects of trial type on target-related activation Three regions showed interactions between trial type (incongruent, neutral, congruent) and time point: one left DLPFC region, one left putamen region, and one left caudate region. According to post-hoc paired $t$ tests at Time Point 4 , these regions displayed greater activation in the target phase on incongruent than on either congruent or neutral trials (see
Table 5 for the exact coordinates of each region, and Fig. 7 for example time courses for each region).

Interactions of reward context and trial type We were also interested in whether reward context interacted with trial type (incongruent, neutral, congruent) as an indicator of whether reward context modulated conflict-related brain activity in DLPFC

Table 3 Transient cue-driven effects

\begin{tabular}{|c|c|c|c|c|c|c|c|c|}
\hline \multirow[t]{2}{*}{ Analysis } & \multirow[t]{2}{*}{ BA } & \multirow[t]{2}{*}{ Region of Activation } & \multirow[t]{2}{*}{ Cluster Size (voxels) } & \multicolumn{3}{|c|}{ Talairach Coordinates } & \multirow[t]{2}{*}{$Z$} & \multirow[t]{2}{*}{ Activation Pattern $^{\mathrm{a}}$} \\
\hline & & & & $x$ & $y$ & $z$ & & \\
\hline \multirow[t]{2}{*}{ DLPFC } & 9 & Middle frontal gyrus & 380 & 37 & 25 & 28 & 4.42 & $\mathrm{RC}>\mathbf{R C X T}=\mathrm{BCXT}$ \\
\hline & 9 & Middle frontal gyrus & 294 & -35 & 25 & 28 & 4.40 & $\mathrm{RC}>\mathbf{R C X T}=\mathbf{B C X T}$ \\
\hline \multirow[t]{4}{*}{ Basal ganglia } & & Lateral globus pallidus & 282 & 17 & 0 & 7 & 4.76 & $\mathrm{RC}>\mathbf{R C X T}=\mathrm{BCXT}$ \\
\hline & & Lateral globus pallidus & 143 & -20 & -5 & 2 & 3.63 & $\mathrm{RC}>\mathrm{RCXT}=\mathbf{B C X T}$ \\
\hline & & Caudate head & 14 & -7 & 3 & 4 & 2.80 & $\mathrm{RC}>\mathbf{R C X T}=\mathbf{B C X T}$ \\
\hline & & Caudate body & 50 & -16 & -5 & 17 & 3.72 & $\mathrm{RC}>\mathbf{R C X T}=\mathrm{BCXT}$ \\
\hline
\end{tabular}

Bold indicates deactivated regions

$B C X T$ baseline context trials, $D L P F C$ dorsolateral prefrontal cortex, $R C$ reward cue trials, $R C X T$ reward context trials

${ }^{a}$ Post-hoc paired $t$ tests were conducted to examine the relationship between neural activity on reward trials and other cue-type activity on each region at Time Point $4(p<.05)$ 


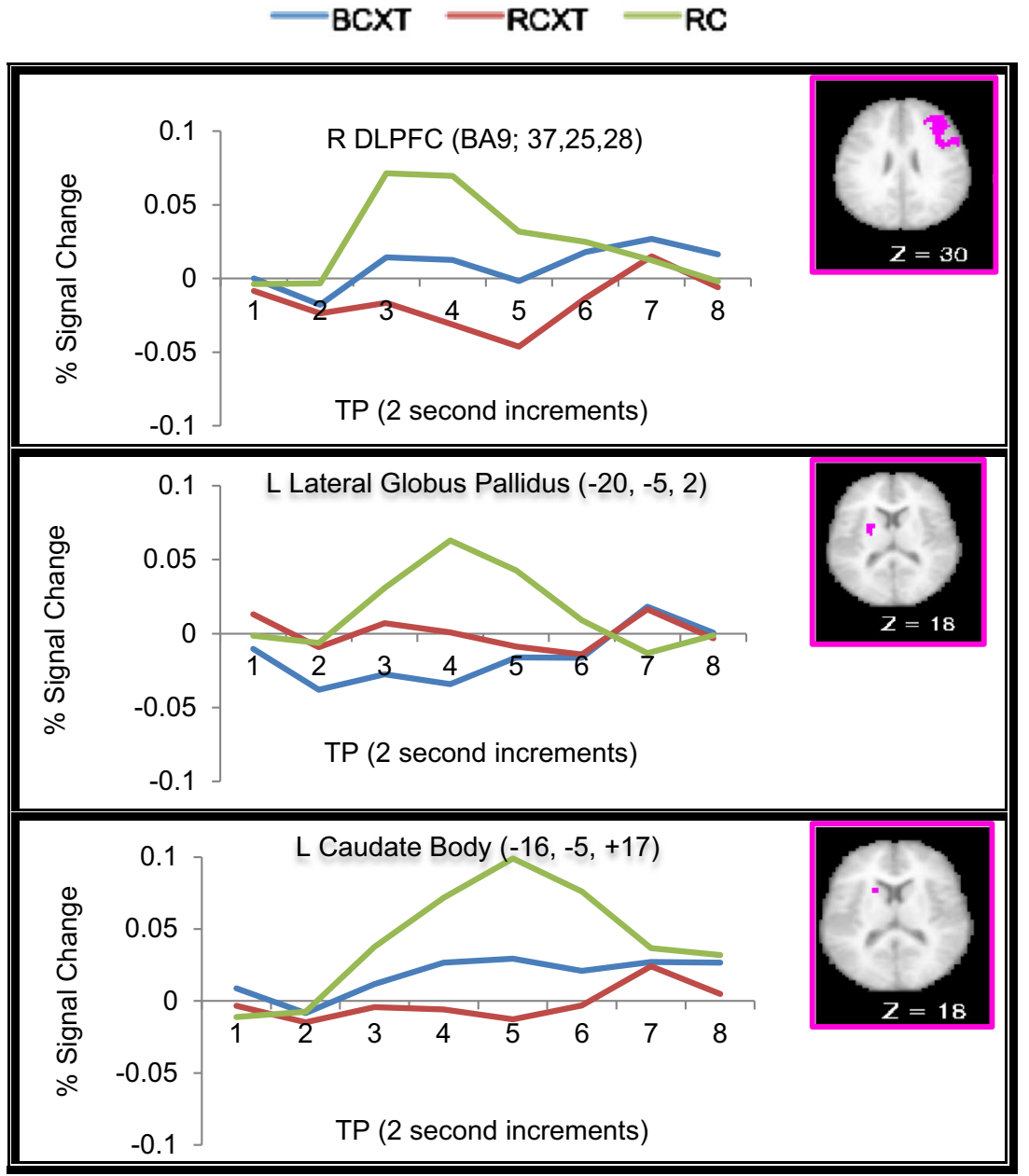

Fig. 4 Example time courses from regions of interest displaying significant interactions of reward and time point. Note: $L$ Left, $R$ Right, $T P$ Time Point

or striatum. No region in the DLPFC or BG mask regions displayed significant Reward Context $\times$ Trial Type interaction.

\section{Discussion}

The goals of the present study were (1) to replicate previous findings regarding sustained context-dependent and transient cue-driven effects of reward on cognitive control in healthy adults, especially by focusing on the involvement of the
DLPFC and the striatum; and (2)to extend this line of research by examining the influence of individual differences in anhedonia. We found sustained increases in brain activations in bilateral DLPFC and in the putamen and caudate as a function of reward context. Furthermore, consistent with prior work, several regions in the bilateral DLPFC and other subcortical regions, including the lateral globus pallidus and the caudate, showed transient increases of brain activations as a function of reward cues. Importantly, individual differences in anhedonia predicted transient neural responses to reward-predicting cues

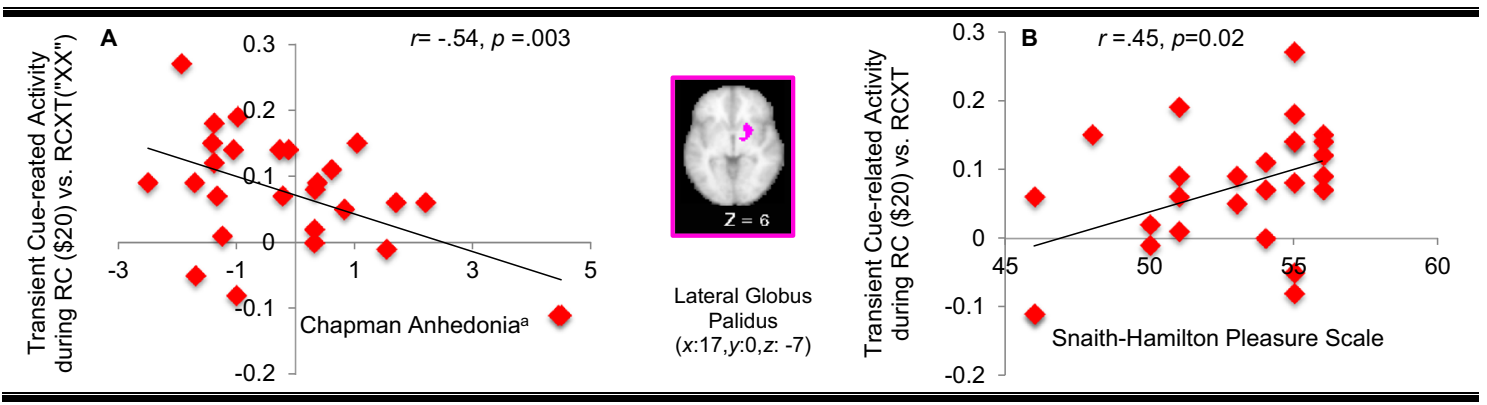

Fig. 5 Significant association between transient components of motivated cognitive control and hedonic tone 
Table 4 Effects from Reward Context (BCXT, RCXT, RC) $\times$ Time Point ANOVAs in the target phase

\begin{tabular}{|c|c|c|c|c|c|c|c|c|}
\hline \multirow[t]{2}{*}{ Analysis } & \multirow[t]{2}{*}{ BA } & \multirow[t]{2}{*}{ Region of Activation } & \multirow[t]{2}{*}{ Cluster Size (voxels) } & \multicolumn{3}{|c|}{ Talairach Coordinates } & \multirow[t]{2}{*}{$Z$} & \multirow[t]{2}{*}{ Activation Pattern ${ }^{\mathrm{a}}$} \\
\hline & & & & $x$ & $y$ & $z$ & & \\
\hline \multirow[t]{2}{*}{ DLPFC } & 9 & Superior frontal gyrus & 36 & 26 & 37 & 29 & 3.39 & $\mathrm{BCXT}>\mathrm{RC}=\mathrm{RCXT}$ \\
\hline & 9 & Middle frontal gyrus & 40 & -33 & 32 & 33 & 3.33 & $\mathrm{BCXT}=\mathrm{RCXT}>\mathrm{RC}$ \\
\hline \multirow[t]{2}{*}{ Basal ganglia } & & Lateral globus pallidus & 55 & -17 & 2 & -3 & 4.71 & $\mathbf{B C X T}=\mathbf{R C X T}>\mathbf{R C}^{\mathrm{c}}$ \\
\hline & & Medial globus pallidus & 26 & 16 & -1 & -4 & 3.82 & $\mathbf{B C X T}=\mathbf{R C X T}>\mathbf{R C}^{\mathrm{c}}$ \\
\hline \multirow[t]{2}{*}{ DLPFC } & 9 & Middle frontal gyrus & 316 & 42 & 18 & 29 & 6.40 & $\mathrm{RC}, \mathrm{RCXT}>\mathrm{BCXT}$ \\
\hline & 9 & Middle frontal gyrus & 137 & -42 & 11 & 30 & 4.24 & $\mathrm{RC}>\mathrm{BCXT}, \mathrm{RCXT}^{\mathrm{b}}$ \\
\hline
\end{tabular}

Bold indicates deactivation

$D L P F C$ dorsolateral prefrontal cortex, $B C X T$ baseline context, $R C$ reward cue, $R C X T$ reward context

${ }^{a}$ Post-hoc paired $t$ tests (BCXT-RC, RC-RCXT, BCXT-RCXT) were conducted at Time Point 4 in the hemodynamic response function (HRF) to identify the sources of significant effects. If significant effects were not observed at Time Point 4 , additional follow-up tests were conducted at other time points in the HRF

b,c Target-related activity at Time Point 4 did not differ as a function of reward-related cue type: $\mathrm{b}$ indicates that a significant effect was observed at Time Point 5, c that a significant effect was observed at Time Point 7

in the lateral globus pallidus. Each of these findings is discussed in more detail below.

\section{Behavioral reward context and cue effects}

The accuracy data showed a main effect of trial type, reflecting more errors on incongruent than on congruent and neutral trials. However, we did not find main effect of reward or a Reward $\times$ Trial Type interaction in the accuracy data, meaning that we did find that incentives modulated accuracy. However, consistent with previous studies showing enhancement of rewards for cognitive function (Chiew \& Braver, 2013; Gilbert \& Fiez, 2004; Hughes, Mathan, \& Yeung, 2013; Locke \& Braver, 2008; Strang \& Pollak, 2014; Vuillier, Whitebread, \& Szucs, 2015), we did find a main effect of reward in the RT data, with faster responses on reward cue trials than on no-reward cue trials in the reward block (incentive cue effect), as well as faster responses on

$-\mathrm{BCXT}-\mathrm{RCXT}-\mathrm{RC}$

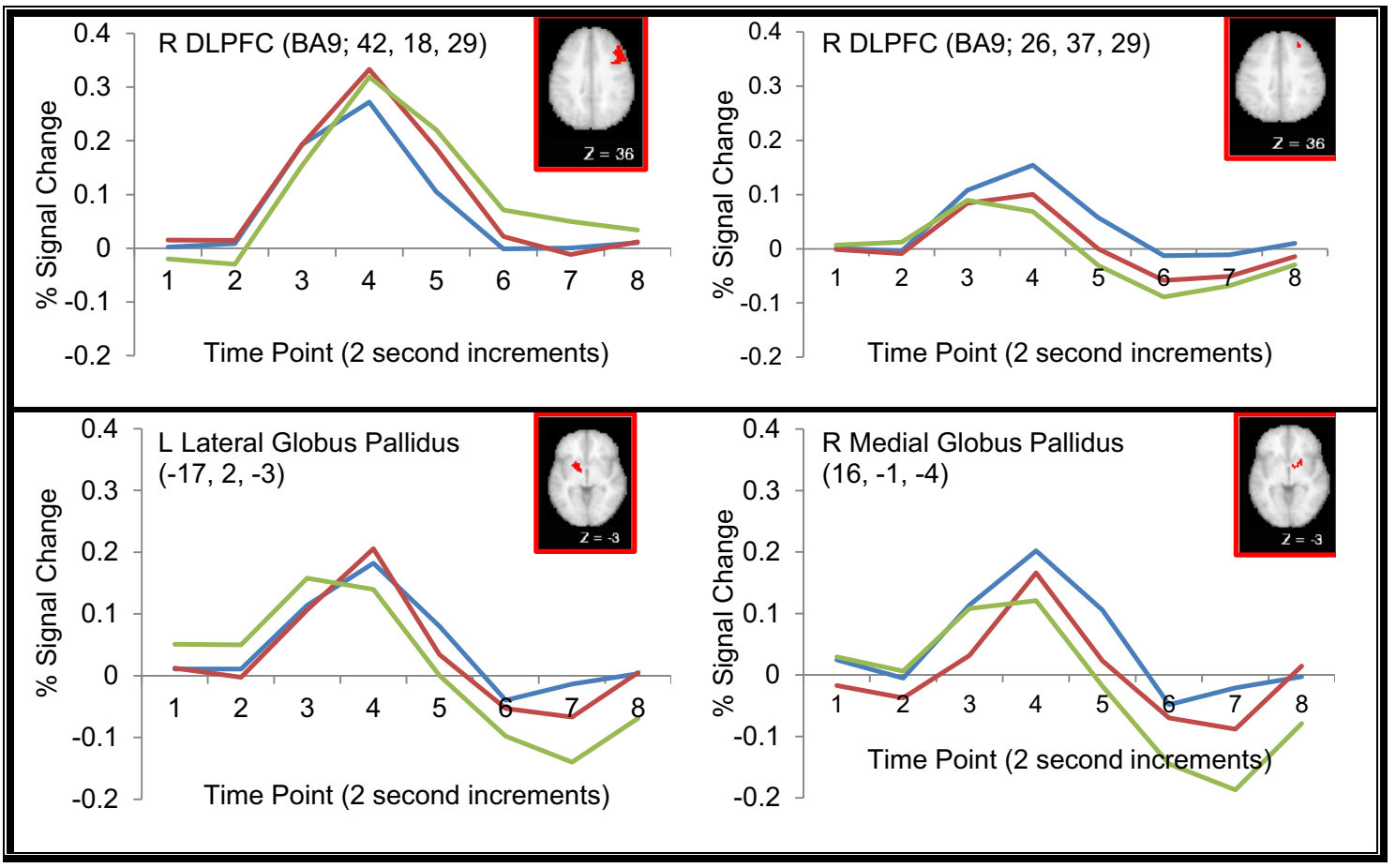

Fig. 6 Example time courses from regions of interest displaying Reward Context $\times$ Time Point interactions in the target phase 
Table 5 Effects from Trial Type $\times$ Time Point ANOVAs in the target phase

\begin{tabular}{|c|c|c|c|c|c|c|c|c|}
\hline \multirow[t]{2}{*}{ Analysis } & \multirow[t]{2}{*}{$\mathrm{BA}$} & \multirow[t]{2}{*}{ Region of Activation } & \multirow[t]{2}{*}{ Cluster Size (voxels) } & \multicolumn{3}{|c|}{ Talairach Coordinates } & \multirow[t]{2}{*}{$Z$} & \multirow[t]{2}{*}{ Activation Pattern $^{\mathrm{a}}$} \\
\hline & & & & $x$ & $y$ & $z$ & & \\
\hline DLPFC & 9 & Middle frontal gyrus & 213 & -42 & 13 & 29 & 4.12 & $\mathrm{I}>\mathrm{C}=\mathrm{N}$ \\
\hline \multirow[t]{2}{*}{ Basal ganglia } & & Putamen & 37 & -20 & 2 & 5 & 3.34 & $\mathrm{I}=\mathrm{C}>\mathrm{N}^{+}$ \\
\hline & & Caudate body & 26 & -11 & 0 & 11 & 2.67 & $\mathrm{I}>\mathrm{C}=\mathrm{N}$ \\
\hline
\end{tabular}

$C$ congruent, $I$ incongruent, $N$ neutral

${ }^{a}$ Post-hoc paired $t$ tests (incongruent-neutral, incongruent-congruent, neutral-congruent) were conducted at Time Point 4 to identify the source of significant effects. If significant effects were not observed at Time Point 4, additional follow-up tests were conducted at other time points in the hemodynamic response function

${ }^{+}$Neural activity at Time Point 4 did not differ depending on the type of trials. A significant effect was observed at Time Point $3, p<.05$

no-reward cue trials in the reward block than in the baseline block (incentive context effect). These enhancing effects of reward on RT suggest that knowledge of potential rewards enhanced participants' behavioral performance, potentially through facilitated representations of reward value within the reward contexts. However, none of these main effects interacted further with trial type.

These results are somewhat in contrast with those of Padmala and Pessoa (2011), from whom we modified the same task paradigm for use with a mixed state-item fMRI design. Padmala and Pessoa found an interaction between reward and congruency, suggesting reduced interference effects (incongruent vs. neutral) in the reward as compared to the no-reward condition. The discrepancy in the magnitudes of the Reward $\times$ Trial Type interaction effect between the present and the previous study (Padmala \& Pessoa, 2011) might reflect the different populations in each study. In the present study, our participants were from the community (mean age: $35.56 \pm 8.61$ years old), whereas those in Padmala and Pessoa's work were college students (mean age: $22 \pm 5$ years old). Although the reward values were similar in the two studies ( $\$ 18$ in Padmala \& Pessoa's work, $\$ 20$ in the present work), it is possible that the subjective reward value of these amounts might not have generated the same

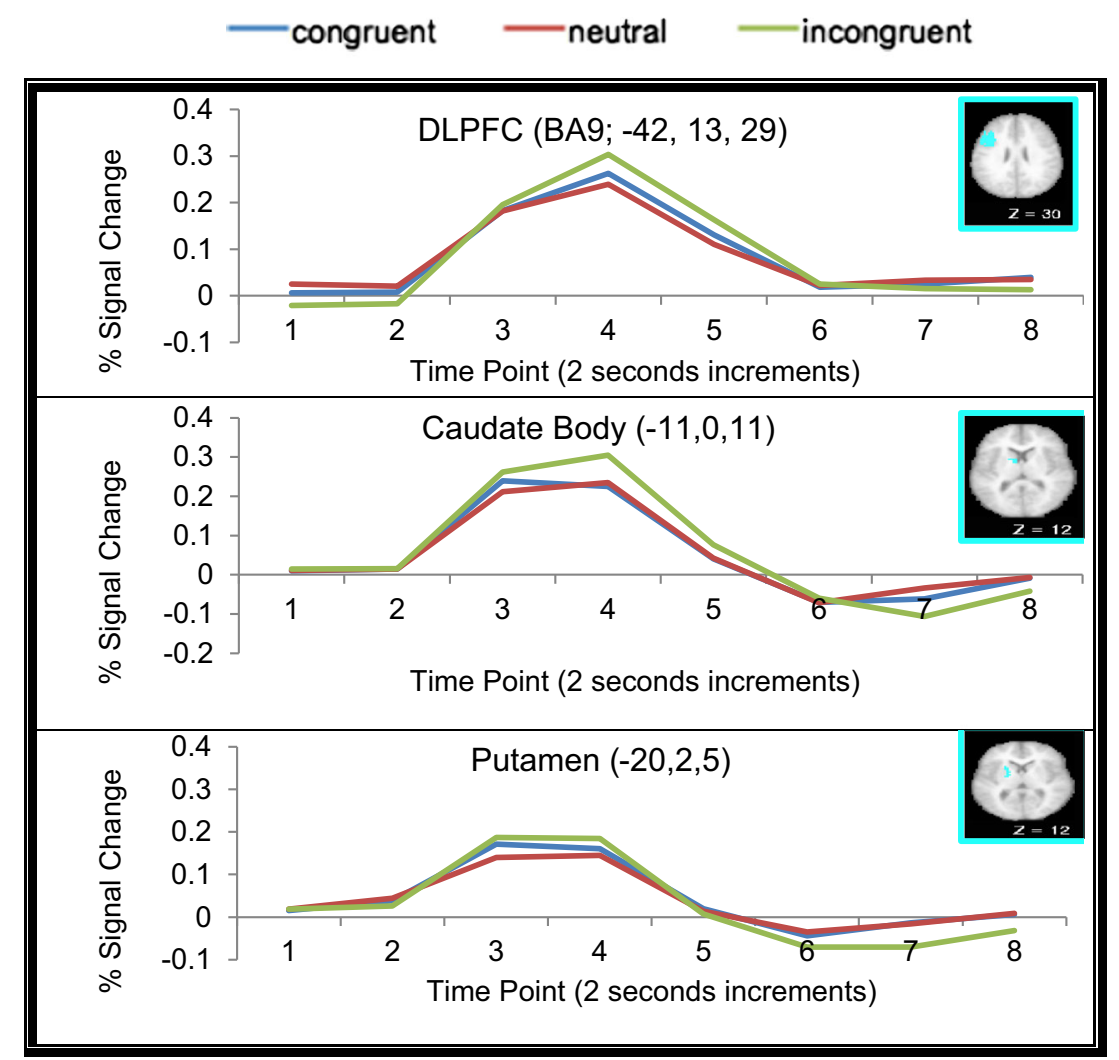

Fig. 7 Time courses from regions of interest displaying Trial Type $\times$ Time Point interactions in the target phase 
level of motivation among older community populations that it did among college students (Padmala \& Pessoa, 2011). Although the participants in the present study clearly sped their responses as a function of reward, it is possible that the reward value among community populations in present study was not as salient enough to actually reduce the magnitude of the conflict effect (Padmala \& Pessoa, 2011). Furthermore, it is possible that the task difficulty in the present study was not sufficiently challenging to leave room for modulation of the conflict effect. Specifically, the accuracy level was somewhat higher in the present study (mean error rates $\sim 3 \%$ ) than in Padmala and Pessoa's (mean error rates $\sim 6 \%$ ). Nonetheless, the fact that participants in the present study were able to speed their responses without sacrificing accuracy suggests that both the reward cues and the reward context were able to enhance task processing, though not to the point of reducing the magnitude of the conflict effect.

\section{Transient cue-related effects of reward-modulated cognitive control}

In the present study, activation was increased in response to incentive cues versus no-incentive cues in bilateral DLPFC and in several reward-related subcortical regions, such as the lateral globus pallidus and caudate. These results are in line with prior work (e.g., Padmala \& Pessoa, 2011; Vassena et al., 2014), which also showed transient increases in a distributed network of several regions, including the lateral PFC and parietal cortex, that are thought to be engaged in cognitive control (Owen, McMillan, Laird, \& Bullmore, 2005; Wager \& Smith, 2003).

The DLPFC has traditionally been considered a core component of cognitive control, as we described in the introduction. Recently, accumulated evidence has suggested that the DLPFC represents reward-related value information as well as "cold" information about task goals (see Dixon \& Christoff, 2014, for a recent review). Furthermore, anatomical evidence has shown that the DLPFC is strongly connected with key regions involved in value representations, such as the orbitofrontal cortex and the anterior cingulate cortex (Pandya, Van Hoesen, \& Mesulam, 1981; Petrides \& Pandya, 1999, 2007). Also, the DLPFC (BA 9/10) projects to the $\mathrm{BG}$, including the caudate nucleus and the globus pallidus. Projections from these striatal regions terminate in the thalamus, which in turn projects back to the DLPFC and to premotor and motor cortices (Joel \& Weiner, 1994, 1997). With regard to this circuit of anatomical connections, the increased DLPFC and striatal activations in response to incentive cues might reflect enhanced neural representations of reward value through a top-down regulation of activations in this circuit.

In addition to the modulation of cue-related responses as a function of incentives, we also found modulation of target- related responses. As we expected on the basis of the prior literature, we found that neural activation on the incongruent trials was greater than the congruent and neutral activations in several regions of the DLPFC, as well as in both a left putamen and a left caudate region. Interestingly, small, bilateral, relatively medial and anterior regions of the DLPFC showed decreased target-related activations in the reward trials as compared to the baseline trials, whereas more lateral portions of the DLPFC showed the opposite pattern of greater targetrelated activations during the $\mathrm{RC}$ trials. The more lateral portions of the DLPFC are those more typically associated with cognitive control. In the Jimura et al. (2010) study, activations in a similar region on the right showed reduced activations at the time of the target, as compared to that of the cue. These researchers interpreted this effect as potentially reflecting decreased use of reactive control, though activations did not differ during the target phase for reward versus nonreward trials. As such, our results might suggest that in middle-aged community controls, reward cues encourage enhanced activations in cognitive-control-related regions, even during task execution. However, we are less clear what the functional significance may be of the moderated activation in the more medial DLPFC regions, and thus further research will be needed to elucidate the implications of these activation differences.

\section{Sustained context-dependent effects of motivated cognitive control}

As we discussed in the introduction, information about incentives can modulate brain activations in both a transient cuerelated fashion and a more sustained fashion. Consistent with these previous findings, our results showed that a number of regions in bilateral DLPFC as well as in bilateral putamen showed greater sustained activations during reward blocks than during the nonreward, baseline blocks. According to the dual-mechanisms-of-control framework, proposed by Braver and his colleagues (Braver, 2012; Braver, Paxton, Locke, \& Barch, 2009), cognitive control is thought to involve two types of control - preparatory, proactive control and reactive control. In prior work, Braver and colleagues argued that incentive cues promote a shift to proactive control, thought to be reflected in both enhanced cue-related activation as well as sustained neural activation in the DLPFC throughout the entire task context. This kind of sustained activation may reflect the active maintenance of task-relevant context information supported by the DLPFC. On the other hand, reactive control is thought to be represented as transient activations supported by a wider brain network, including the DLPFC, parietal cortex, and other reward-related subcortical regions, based on cognitive demand. In line with this hypothesis, the increased neural activation supported by the DLPFC during reward contexts in the present study may have reflected actively 
maintained reward-related context information, potentially reflecting enhanced proactive control.

Several regions in the BG also showed greater sustained activity during reward contexts. The present results extend prior work by suggesting that regions in a fronto-parietal network can show sustained increases with regard to reward information. For example, both Jimura et al. (2010) and Locke and Braver (2008) found sustained increases in the parietal cortex as well as the lateral PFC during reward contexts, but did not find effects in the BG. However, in the present study we conducted a hypothesis-driven analysis using an a priori ROI approach, whereas the previous studies had used voxelwise whole-brain analysis. It is possible that this ROI approach offered greater power to detect sustained effects in both prefrontal and striatal regions (Nieto-Castanon, Ghosh, Tourville, \& Guenther, 2003). This sustained activity may be reflective of BG's hypothesized role in reward-based learning and goal-directed behavior (Dasgupta, Worgotter, \& Manoonpong, 2014; Schultz, Tremblay, \& Hollerman, 1998). Especially, the dorsal striatum is known to receive extensive projections from the DLPFC as well as other frontal regions. Thus, increased sustained activity in the dorsal striatum during the reward context may have represented greater effort to maintain reward-related context information, which may facilitate preparatory responses throughout reward contexts.

\section{Implications for the dual-mechanisms-of-control framework in the reward context}

As we described in introduction, according to the dualmechanisms-of-control model, cognitive control operates via proactive and reactive control modes in different timescales, along with specific task demands. Proactive control refers to sustained and anticipatory processing of control, whereas reactive control refers to stimulus-driven, transient adjustment of control. Therefore, we hypothesized that proactive control would operate during both periods transient updating processing - during the preparatory, anticipatory period prior to stimulus onset, as well as throughout the entire task condition, due to stable task maintenance. On the other hand, reactive control should operate relatively late in the trial and should be more apparent at the time of the response to the probe. In the present study, as we present in the supplementary information, several regions in bilateral DLPFC displayed both sustained activations during reward contexts and transient cue-related activations. We interpret these overlapping regions as a reflection of a proactive control mode for stable and preparatory maintenance of reward-related context information in reward contexts. Also, we found that more lateral portions of the DLPFC showed greater activation on RC than on BCXT trials during the target phase, which may reflect transient reactive control. During the target phase, reactive control may reaccess task-related goal information on a trial-by-trial basis.

These results are consistent with previous work suggesting reward-dependent temporal shifts in DLPFC activations from a transient to a tonic mode during reward contexts (Jimura et al., 2010; Soutschek, Stelzel, Paschke, Walter, \& Schubert, 2015; Strang \& Pollak, 2014; Wilk, Ezekiel, \& Morton, 2012). For example, Jimura et al. (2010) found the involvement of the DLPFC (BA46/9) during both sustained effects through the entire reward conditions, and early-trial components of transient responses at cue presentation. Interestingly, increased sustained activations in the DLPFC during reward versus no-reward contexts lead to a reduction of transient activation during the target phase, potentially suggesting a distinction between proactive mechanisms throughout the entire task block and reactive control mechanisms operating transiently during the later target phase. Consistent with these data, in other work Wilk et al. (2012) asked healthy participants to perform a size congruency task in which two digits that differed in physical and numerical magnitude were presented, and the job of this task was to select the numerically larger digit. Consistent with the present findings, Wilk et al. (2012) found transient, moment-to-moment adjustments of reactive control in bilateral DLPFC and anterior cingulate cortex during target responses, and increased sustained, stable task-set maintenance in several regions in the superior frontal gyrus. Taken together, our findings suggest that motivational incentives influence cognitive control function via sustained and anticipatory proactive control, thought to enhance reward value representations, and also via transient adjustments of reactive control, potentially by updating task-relevant goal information on a trial-by-trial basis for successful response selection. These proactive and reactive control modes appear to activate in distinct temporal modes, but at least partially overlap anatomically in the DLPFC and BG (van Belle, Vink, Durston, \& Zandbelt, 2014).

\section{Relationships to anhedonia}

In addition to group effects of reward-modulated cognitive control, the present study revealed that individual variations in anhedonia were associated with transient reward cuerelated activations in the lateral globus pallidus, but not with sustained context-dependent activation in the DLPFC or BG. The lateral globus pallidus is the final output site of the BG (Joel \& Weiner, 1994). Studies in nonhuman monkeys have revealed that neural activations in the BG are modulated by expected rewards (Hikosaka, Nakamura, \& Nakahara, 2006). For example, in Hong and Hikosaka (2008), monkeys performed a saccade task in which a visual target was presented randomly on the left or the right. One direction was associated with getting rewards, whereas the other predicted no-reward outcomes. When the researchers measured the firing activity 
in the globus pallidus and lateral habenula, they found rewarddependent modulations of neural activity in the globus pallidus, as evidenced by increased activity in response to the reward-predicting target, and decreased responses to the no-reward-predicting target.

In the present study, we found that people reporting greater trait anhedonia showed less neural activation as a function of reward-predicting cues, but that there was no significant association between anhedonia and sustained context-dependent activation during reward contexts. These results are consistent with the hypothesis that anhedonia, or reductions in the ability to experience pleasure, may influence the degree to which the experience of rewards (or "liking") influences modulations of brain activation or behavior in response to the explicit presentation of reward cues, as occurred on RC trials. However, these results do not support the idea that anhedonia influences more global effects of incentives that are reflected in either the sustained aspects of brain activation or the behavioral effects of reward context. As we discussed in the introduction, without having positive experiences/expectations of rewards, it may be hard to exert the effort to pursue goal-directed behavior, potentially providing one mechanism by which individual differences in anhedonia may influence behavior.

\section{Limitations and future directions}

Although this study provides crucial insight into one potential neural mechanism underlying anhedonia, it has several limitations that may be answered in future studies. One limitation to this study is that we did not find significant reductions in conflict effects in either accuracy or RT, though we did find a speeding of RT as a function of reward, without a sacrifice in accuracy. Consistent with these behavioral findings, we did not find significant regions displaying interaction effects of reward context and trial type in the target phase, though our analyses were focused on the DLPFC and the basal ganglia. As we discussed above, we cannot exclude a possibility that the difficulty level of present task may not have been optimally adjusted to tap cognitive control among community populations. Future research varying the difficulty level of task performance is needed to understand how engagement of the DLPFC to modulate cognitive control in reward contexts is moderated by the challenge level of the tasks. In summary, the present findings show both sustained context-dependent and transient cue-driven effects of reward on cognitive control thought to be supported by the DLPFC and the basal ganglia. Importantly, self-reported anhedonia trait in healthy adults were associated with transient neural activity during reward predictions implicated in the lateral globus pallidus, but not with sustained DLPFC activity.

Relevant to the present findings of no interaction between reward and trial type in the behavioral data, there is a possibility that our smaller sample size $(n=27)$ than in previous work by Padmala and Pessoa $(2011 ; n=45)$, whose task paradigm we modified and used in the present study, may have resulted in a failure to replicate some aspects of their work. However, an $N$ of 27 still provided a reasonable power to detect transient and sustained effects of interest in the present study and the correlation coefficients ranging from .4 to .5 that are used for individual-difference analyses. Importantly, when we computed effect sizes for the enhancement of rewards on RT performance, the present study yielded an effect size for RT data $(d=0.33)$ similar to that from Padmala and Pessoa $(d=0.37)$.

Another major limitation is that, due to the fixed-order presentation of the baseline and reward conditions, the sustained context-dependent effect might have reflected practice-related effects. However, our differences between reward conditions are unlikely to have been due to practice effects, given the previous empirical evidence against this possibility. For example, Chiew and Braver (2013) examined the effect of reward incentives on cognitive control, as measured by the AX-continuous performance task (AX-CPT). Like us, they utilized a mixed block/event design. In the AX-CPT, in which participants perform baseline (no incentive offered) and reward blocks, within the reward blocks, nonincentive trials are randomly intermixed with the incentive trials. In Chiew and Braver's supplementary analysis, they broke down each block into four 50-trial epochs, and found that potential practice effects disappeared after the first epoch, whereas incentive effects remained throughout. Furthermore, the differences between our RCXT and RC trials cannot be due to practice effects, since these trials were interleaved.

The present mixed state-item design allowed us to start to dissociate sustained versus transient effects of rewards. However, additional approaches could be used in future studies to further dissociate these effects. In particular, our design did not provide an estimate of the effects of sustained rewards in the absence of transient trial-by-trial effects. Thus, an alternative future design would be to include conditions in which participants were informed that there would be a bonus at the end of a block of trials for overall improved performance, but no trial-specific reward cues. With such a manipulation, one could create a crossed design in which there either was or was not an overall block-wise manipulation of reward bonus (e.g., context effect: high vs. low sustained reward motivation) versus the presence or absence of trial-by-trial cues about the potentially of additional rewards (transient effect: reward cue vs. no-reward cue trials).

In summary, the present findings replicated previous findings showing both sustained context-dependent and transient cue-driven effect modulations of activation in DLPFC and BG regions as a function of reward, thought to reflect modulation of cognitive control. Furthermore, we extended prior research by showing that individual differences in self-reported anhedonia were associated with individual differences in cue- 
related functional brain activation on reward trials in the lateral globus pallidus, but not with sustained DLPFC activation. These individual-difference relationships may reflect the degree to which the hedonic experience of reward influences motivated behavior.

Author note This work was supported by National Institute of Mental Health Grant Number R01-MH066031. All authors have no financial interests or potential conflicts of interest. We thank the members of the Cognitive Control and Psychopathology Laboratory, and all participants in this study who have provided time and effort to make this study possible.

\section{References}

Aupperle, R. L., Melrose, A. J., Francisco, A., Paulus, M. P., \& Stein, M. B. (2015). Neural substrates of approach-avoidance conflict decision-making. Human Brain Mapping, 36, 449-462. doi:10.1002/ hbm.22639

Beaver, J. D., Lawrence, A. D., van Ditzhuijzen, J., Davis, M. H., Woods, A., \& Calder, A. J. (2006). Individual differences in reward drive predict neural responses to images of food. Journal of Neuroscience, 26, 5160-5166. doi:10.1523/jneurosci.0350-06.2006

Boehler, C. N., Hopf, J. M., Krebs, R. M., Stoppel, C. M., Schoenfeld, M. A., Heinze, H. J., \& Noesselt, T. (2011). Task-load-dependent activation of dopaminergic midbrain areas in the absence of reward. Journal of Neuroscience, 31, 4955-4961. doi:10.1523/jneurosci. 4845-10.2011

Boehler, C. N., Schevernels, H., Hopf, J. M., Stoppel, C. M., \& Krebs, R. M. (2014). Reward prospect rapidly speeds up response inhibition via reactive control. Cognitive, Affective, \& Behavioral Neuroscience, 14, 593-609. doi:10.3758/s13415-014-0251-5

Braver, T. S. (2012). The variable nature of cognitive control: A dual mechanisms framework. Trends in Cognitive Sciences, 16, 106113. doi:10.1016/j.tics.2011.12.010

Braver, T. S., Gray, J. R., \& Burgess, G. C. (2007). Explaining the many varieties of working memory variation: Dual mechanisms of cognitive control. In A. R. A. Conway, C. Jarrold, M. J. Kane, A. Miyake, \& J. N. Towse (Eds.), Variation in working memory (pp. 76-106). New York: Oxford University Press.

Braver, T. S., Krug, M. K., Chiew, K. S., Kool, W., Westbrook, J. A., Clement, N. J., \& Somerville, L. H. (2014). Mechanisms of motivation-cognition interaction: Challenges and opportunities. Cognitive, Affective, \& Behavioral Neuroscience, 14, 443-472. doi:10.3758/ s13415-014-0300-0

Braver, T. S., Paxton, J. L., Locke, H. S., \& Barch, D. M. (2009). Flexible neural mechanisms of cognitive control within human prefrontal cortex. Proceedings of the National Academy of Sciences, 106, 7351-7356. doi:10.1073/pnas.0808187106

Breiter, H. C., \& Rosen, B. R. (1999). Functional magnetic resonance imaging of brain reward circuitry in the human. Annuals of the New York Academy of Sciences, 877, 523-547.

Buckner, R. L., Head, D., Parker, J., Fotenos, A. F., Marcus, D., Morris, J. C., \& Snyder, A. Z. (2004). A unified approach for morphometric and functional data analysis in young, old, and demented adults using automated atlas-based head size normalization: Reliability and validation against manual measurement of total intracranial volume. NeuroImage, 23, 724-738. doi:10.1016/j.neuroimage.2004. 06.018

Chapman, J. P., Chapman, L. J., \& Kwapil, T. R. (1995). Scales for the measurement of schizotypy. In T. Raine, T. Lencz, \& S. Mednick
(Eds.), Schizotypal personality (pp. 79-106). New York: Cambridge University Press.

Chapman, L. J., \& Chapman, J. P. (1978). The revised physical anhedonia scale. Madison: University of Wisconsin.

Chiew, K. S., \& Braver, T. S. (2013). Temporal dynamics of motivationcognitive control interactions revealed by high-resolution pupillometry. Frontiers in Psychology, 4, 15. doi:10.3389/fpsyg. 2013.00015

Cohen, M. X., Young, J., Baek, J. M., Kessler, C., \& Ranganath, C. (2005). Individual differences in extraversion and dopamine genetics predict neural reward responses. Brain Research Cognitive Brain Research, 25, 851-861. doi:10.1016/j.cogbrainres.2005.09.018

Cooper, A. J., Duke, E., Pickering, A. D., \& Smillie, L. D. (2014). Individual differences in reward prediction error: contrasting relations between feedback-related negativity and trait measures of reward sensitivity, impulsivity and extraversion. Frontiers in Human Neuroscience, 8, 248. doi:10.3389/fnhum.2014.00248

Dasgupta, S., Worgotter, F., \& Manoonpong, P. (2014). Neuromodulatory adaptive combination of correlation-based learning in cerebellum and reward-based learning in basal ganglia for goal-directed behavior control. Frontiers in Neural Circuits, 8, 126. doi:10.3389/fncir. 2014.00126

Dixon, M. L., \& Christoff, K. (2012). The decision to engage cognitive control is driven by expected reward-value: Neural and behavioral evidence. PLoS ONE, 7, e51637. doi:10.1371/journal.pone. 0051637

Dixon, M. L., \& Christoff, K. (2014). The lateral prefrontal cortex and complex value-based learning and decision making. Neuroscience \& Biobehavioral Reviews, 45, 9-18. doi:10.1016/j.neubiorev.2014. 04.011

Eckblad, M., Chapman, L. J., Chapman, J. P., \& Mishlove, M. (1982). The revised social anhedonia scale. Madison: University of Wisconsin.

Engelmann, J. B., Damaraju, E., Padmala, S., \& Pessoa, L. (2009). Combined effects of attention and motivation on visual task performance: Transient and sustained motivational effects. Frontiers in Human Neuroscience, 3, 4. doi:10.3389/neuro.09.004.2009

Fischl, B., Salat, D. H., Busa, E., Albert, M., Dieterich, M., Haselgrove, C., \& Dale, A. M. (2002). Whole brain segmentation: automated labeling of neuroanatomical structures in the human brain. Neuron, 33, 341-355.

Franken, I. H., Rassin, E., \& Muris, P. (2007). The assessment of anhedonia in clinical and non-clinical populations: Further validation of the snaith-Hamilton pleasure scale (SHAPS). Journal of Affective Disorders, 99, 83-89. doi:10.1016/j.jad.2006.08.020

Gilbert, A. M., \& Fiez, J. A. (2004). Integrating rewards and cognition in the frontal cortex. Cognitive, Affective, \& Behavioral Neuroscience, 4, 540-552. doi:10.3758/CABN.4.4.540

Gorwood, P. (2008). Neurobiological mechanisms of anhedonia. Dialogues in Clinical Neuroscience, 10, 291-299.

Gradin, V. B., Kumar, P., Waiter, G., Ahearn, T., Stickle, C., Milders, M., $\&$ Steele, J. D. (2011). Expected value and prediction error abnormalities in depression and schizophrenia. Brain, 134, 1751-1764. doi:10.1093/brain/awr059

Harvey, P. O., Pruessner, J., Czechowska, Y., \& Lepage, M. (2007). Individual differences in trait anhedonia: A structural and functional magnetic resonance imaging study in non-clinical subjects. Molecular Psychiatry, 12(703), 767-775. doi:10.1038/sj.mp. 4002021

Hikosaka, O., Nakamura, K., \& Nakahara, H. (2006). Basal ganglia orient eyes to reward. Journal of Neurophysiology, 95, 567-584. doi:10. $1152 /$ jn.00458.2005

Hollerman, J. R., Tremblay, L., \& Schultz, W. (2000). Involvement of basal ganglia and orbitofrontal cortex in goal-directed behavior. Progress in Brain Research, 126, 193-215. doi:10.1016/S00796123(00)26015-9 
Hong, S., \& Hikosaka, O. (2008). The globus pallidus sends rewardrelated signals to the lateral habenula. Neuron, 60, 720-729. doi: 10.1016/j.neuron.2008.09.035

Hughes, G., Mathan, S., \& Yeung, N. (2013). EEG indices of reward motivation and target detectability in a rapid visual detection task. NeuroImage, 64, 590-600. doi:10.1016/j.neuroimage.2012.09.003

Ivanov, I., Liu, X., Clerkin, S., Schulz, K., Friston, K., Newcorn, J. H., \& Fan, J. (2012). Effects of motivation on reward and attentional networks: An fMRI study. Brain and Behavior, 2, 741-753. doi:10. $1002 /$ brb3.80

Jimura, K., Locke, H. S., \& Braver, T. S. (2010). Prefrontal cortex mediation of cognitive enhancement in rewarding motivational contexts. Proceedings of the National Academy of Sciences, 107, 8871-8876.

Joel, D., \& Weiner, I. (1994). The organization of the basal gangliathalamocortical circuits: Open interconnected rather than closed segregated. Neuroscience, 63, 363-379.

Joel, D., \& Weiner, I. (1997). The connections of the primate subthalamic nucleus: Indirect pathways and the open-interconnected scheme of basal ganglia-thalamocortical circuitry. Brain Research Reviews, 23, 62-78.

Koch, K., Schachtzabel, C., Wagner, G., Schikora, J., Schultz, C., Reichenbach, J. R., \& Schlosser, R. G. (2010). Altered activation in association with reward-related trial-and-error learning in patients with schizophrenia. NeuroImage, 50, 223-232. doi:10.1016/j. neuroimage.2009.12.031

Kouneiher, F., Charron, S., \& Koechlin, E. (2009). Motivation and cognitive control in the human prefrontal cortex. Nature Neuroscience, 12, 939-945. doi:10.1038/nn.2321

Krebs, R. M., Boehler, C. N., Appelbaum, L. G., \& Woldorff, M. G. (2013). Reward associations reduce behavioral interference by changing the temporal dynamics of conflict processing. PLoS ONE, 8, e53894. doi:10.1371/journal.pone.0053894

Krebs, R. M., Boehler, C. N., Roberts, K. C., Song, A. W., \& Woldorff, M. G. (2012). The involvement of the dopaminergic midbrain and cortico-striatal-thalamic circuits in the integration of reward prospect and attentional task demands. Cerebral Cortex, 22, 607-615. doi:10. 1093/cercor/bhr134

Krebs, R. M., Boehler, C. N., \& Woldorff, M. G. (2010). The influence of reward associations on conflict processing in the Stroop task. Cognition, 117, 341-347. doi:10.1016/j.cognition.2010.08.018

Locke, H. S., \& Braver, T. S. (2008). Motivational influences on cognitive control: Behavior, brain activation, and individual differences. Cognitive, Affective, \& Behavioral Neuroscience, 8, 99-112. doi:10. 3758/CABN.8.1.99

Manoach, D. S., Greve, D. N., Lindgren, K. A., \& Dale, A. M. (2003). Identifying regional activity associated with temporally separated components of working memory using event-related functional MRI. NeuroImage, 20, 1670-1684.

Miezin, F. M., Maccotta, L., Ollinger, J. M., Petersen, S. E., \& Buckner, R. L. (2000). Characterizing the hemodynamic response: Effects of presentation rate, sampling procedure, and the possibility of ordering brain activity based on relative timing. NeuroImage, 11, 735759. doi:10.1006/nimg. 2000.0568

Nieto-Castanon, A., Ghosh, S. S., Tourville, J. A., \& Guenther, F. H. (2003). Region of interest based analysis of functional imaging data. NeuroImage, 19, 1303-1316.

Niv, Y. (2007). Cost, benefit, tonic, phasic: What do response rates tell us about dopamine and motivation? Annuals of the New York Academy of Sciences, 1104, 357-376. doi:10.1196/annals.1390.018

O’Doherty, J. P. (2004). Reward representations and reward-related learning in the human brain: Insights from neuroimaging. Current Opinion in Neurobiology, 14, 769-776.

Ojemann, J. G., Akbudak, E., Snyder, A. Z., McKinstry, R. C., Raichle, M. E., \& Conturo, T. E. (1997). Anatomic localization and quantitative analysis of gradient refocused echo-planar fMRI susceptibility artifacts. NeuroImage, 6, 156-167.
Ollinger, J. M., Corbetta, M., \& Shulman, G. L. (2001a). Separating processes within a trial in event-related functional MRI. NeuroImage, 13, 218-229. doi:10.1006/nimg.2000.0711

Ollinger, J. M., Shulman, G. L., \& Corbetta, M. (2001b). Separating processes within a trial in event-related functional MRI. Neurolmage, 13, 210-217. doi:10.1006/nimg.2000.0710

Owen, A. M., McMillan, K. M., Laird, A. R., \& Bullmore, E. (2005). Nback working memory paradigm: A meta-analysis of normative functional neuroimaging studies. Human Brain Mapping, 25, 4659.

Padmala, S., \& Pessoa, L. (2011). Reward reduces conflict by enhancing attentional control and biasing visual cortical processing. Journal of Cognitive Neuroscience, 23, 3419-3432. doi:10.1162/jocn a 00011

Pandya, D. N., Van Hoesen, G. W., \& Mesulam, M. M. (1981). Efferent connections of the cingulate gyrus in the rhesus monkey. Experimental Brain Resesarch, 42, 319-330.

Petersen, S. E., \& Dubis, J. W. (2012). The mixed block/event-related design. NeuroImage, 62, 1177-1184. doi:10.1016/j.neuroimage. 2011.09.084

Petrides, M., \& Pandya, D. N. (1999). Dorsolateral prefrontal cortex: Comparative cytoarchitectonic analysis in the human and the macaque brain and corticocortical connection patterns. European Journal of Neuroscience, 11, 1011-1036.

Petrides, M., \& Pandya, D. N. (2007). Efferent association pathways from the rostral prefrontal cortex in the macaque monkey. Journal of Neuroscience, 27, 11573-11586. doi:10.1523/jneurosci.2419-07.2007

Pochon, J.B., Levy, R., Fossati, P., Lehericy, S., Poline, J.B., Pillon, B., ... Dubois, B. (2002). The neural system that bridges reward and cognition in humans: An fMRI study. Proceedings of the National Academy of Sciences, 99, 5669-5674.

Rajkowska, G., \& Goldman-Rakic, P. S. (1995). Cytoarchitectonic definition of prefrontal areas in the normal human cortex: II. Variability in locations of areas 9 and 46 and relationship to the Talairach Coordinate System. Cerebral Cortex, 5, 323-337.

Rothkirch, M., Schmack, K., Deserno, L., Darmohray, D., \& Sterzer, P. (2014). Attentional modulation of reward processing in the human brain. Human Brain Mapping, 35, 3036-3051. doi:10.1002/hbm.22383

Sakagami, M., \& Watanabe, M. (2007). Integration of cognitive and motivational information in the primate lateral prefrontal cortex. Annuals of the New York Academy of Sciences, 1104, 89-107.

Schultz, W., Tremblay, L., \& Hollerman, J. R. (1998). Reward prediction in primate basal ganglia and frontal cortex. Neuropharmacology, 37, 421-429.

Small, D. M., Gitelman, D., Simmons, K., Bloise, S. M., Parrish, T., \& Mesulam, M. M. (2005). Monetary incentives enhance processing in brain regions mediating top-down control of attention. Cerebral Cortex, 15, 1855-1865.

Snaith, R. P., Hamilton, M., Morley, S., Humayan, A., Hargreaves, D., \& Trigwell, P. (1995). A scale for the assessment of hedonic tone the Snaith-Hamilton Pleasure Scale. British Journal of Psychiatry, 167, 99-103.

Soutschek, A., Stelzel, C., Paschke, L., Walter, H., \& Schubert, T. (2015). Dissociable effects of motivation and expectancy on conflict processing: An FMRI study. Journal of Cognitive Neuroscience, 27, 409-423. doi:10.1162/jocn a 00712

Stoppel, C. M., Boehler, C. N., Strumpf, H., Heinze, H. J., Hopf, J. M., \& Schoenfeld, M. A. (2011). Neural processing of reward magnitude under varying attentional demands. Brain Research, 1383, 218-229. doi:10.1016/j.brainres.2011.01.095

Strang, N. M., \& Pollak, S. D. (2014). Developmental continuity in reward-related enhancement of cognitive control. Developmental Cognitive Neuroscience, 10, 34-43. doi:10.1016/j.den.2014.07.005

Talairach, J., \& Tournoux, P. (1988). Co-planar stereotaxic atlas of the human brain: 3-dimensional proportional system. An approach to cerebral imaging. New York: Thieme. 
Taylor, S. F., Welsh, R. C., Wager, T. D., Phan, K. L., Fitzgerald, K. D., \& Gehring, W. J. (2004). A functional neuroimaging study of motivation and executive function. NeuroImage, 21, 1045-1054. doi:10. 1016/j.neuroimage.2003.10.032

van Belle, J., Vink, M., Durston, S., \& Zandbelt, B. B. (2014). Common and unique neural networks for proactive and reactive response inhibition revealed by independent component analysis of functional MRI data. NeuroImage, 103, 65-74. doi:10.1016/j.neuroimage. 2014.09.014

Vassena, E., Silvetti, M., Boehler, C. N., Achten, E., Fias, W., \& Verguts, T. (2014). Overlapping neural systems represent cognitive effort and reward anticipation. PLOS ONE, 9, e91008. doi:10.1371/journal. pone.0091008

Visscher, K. M., Miezin, F. M., Kelly, J. E., Buckner, R. L., Donaldson, D. I., McAvoy, M. P., \& Petersen, S. E. (2003). Mixed blocked/ event-related designs separate transient and sustained activity in fMRI. NeuroImage, 19, 1694-1708.

Vuillier, L., Whitebread, D., \& Szucs, D. (2015). ERP evidence of cognitive strategy change in motivational conditions with varying level of difficulty. Neuropsychologia, 70, 126-133. doi:10.1016/j. neuropsychologia.2015.02.025
Wager, T. D., \& Smith, E. E. (2003). Neuroimaging studies of working memory: A meta-analysis. Cognitive, Affective, \& Behavioral Neuroscience, 3, 255-274.

Wang, L., Mamah, D., Harms, M. P., Karnik, M., Price, J. L., Gado, M. H., \& Csernansky, J. G. (2008). Progressive deformation of deep brain nuclei and hippocampal-amygdala formation in schizophrenia. Biological Psychiatry, 64, 1060-1068. doi:10.1016/j.biopsych. 2008.08.007

Watanabe, M. (2007). Role of anticipated reward in cognitive behavioral control. Current Opinion in Neurobiology, 17, 213-219. doi:10. 1016/j.conb.2007.02.007

Watanabe, M., \& Sakagami, M. (2007). Integration of cognitive and motivational context information in the primate prefrontal cortex. Cerebral Cortex, 17(Suppl. 1), 101-109. doi:10.1093/cercor/ bhm067

Wilk, H. A., Ezekiel, F., \& Morton, J. B. (2012). Brain regions associated with moment-to-moment adjustments in control and stable task-set maintenance. NeuroImage, 59, 1960-1967. doi:10.1016/j. neuroimage.2011.09.011

Woods, R. P., Cherry, S. R., \& Mazziotta, J. C. (1992). Rapid automated algorithm for aligning and reslicing PET images. Journal of Computer Assisted Tomography, 16, 620-633. 